\title{
Stress calculations on multiply connected domains
}

\author{
Johan Helsing $^{\dagger}$ and Anders Jonsson ${ }^{\ddagger}$ \\ ${ }^{\dagger}$ Numerical Analysis, Center for Mathematical Sciences, Lund University, Box 118, S-221 00 Lund, \\ Sweden; and ${ }^{\ddagger}$ Department of Solid Mechanics, Royal Institute of Technology, S-100 44 Stockholm, \\ Sweden.
}

The outstanding problem of finding a simple Muskhelishvili-type integral equation for stress problems on multiply connected domains is solved. Complex potentials are represented in a way which allows for the incorporation of cracks and inclusions. Several numerical examples demonstrate the generality and extreme stability of the approach. The stress field is resolved with a relative error less than $10^{-10}$ on a large, yet simply reproducible, setup with a loaded square plate containing 4096 holes and cracks. Comparison with previous results in the literature indicates that general purpose finite element software may perform better than many special purpose codes.

Key Words: multiply connected domain, cracks, holes, stress concentration factor, stress intensity factor, Fredholm integral equation, fast multipole method

\section{INTRODUCTION}

The problem of solving the equations of linear elasticity on 2D multiply connected domains has received attention for almost a century. Early work dealt with formulations of integral equations and analytical solutions. See the textbooks of Mikhlin [29], Muskhelishvili [30], and Sokolnikoff [39]. Later work focus more on specialized numerical techniques. See Refs [3, 4, 5, 6, 18, 27, 43] for recent examples. Parallel to this development there has been a steady improvement of general purpose finite element packages which, among other things, can solve for stress on multiply connected domains. There has also been progress on fast solvers for large-scale problems [8].

Despite the apparent simplicity of the stress problem and the advances mentioned above, numerical results in the literature are not always correct. There is yet no code which combines error control, rapid execution, and sufficient flexibility as to accurately solve for stress in common setups such as rectangular plates with a large number of cracks, holes, and inclusions. There may be many reasons for this. Stress fields in corners and around crack tips have complicated asymptotic shapes. Special basis functions are needed for good resolution. Further, integral equation based approaches are burdened with

This work was supported by NFR, TFR, and The Knut and Alice Wallenberg Foundation under TFR contracts 98-568 and 99-380. 
some confusion regarding the theory. There are different opinions about what potential representations and equations are the best in different situations. Classic authors [29,30,39] recommend an approach based on the Airy stress function and the Sherman-Lauricella representation. They argue against the Muskhelishvili representation, despite its efficiency for crack problems. Some modern authors, too, prefer to work with the Airy stress function and use real or complex variables and a variety of representations and equations $[4,8$, $11,40,41]$. Others prefer primitive variables [22, 27, 31] or a mix of variables [3] or techniques [26]. Some authors prefer hypersingular integral equations [32, 37].

This paper presents a rapid, stable, and flexible algorithm for the stress problem on multiply connected domains. The algorithm is based on a Fredholm second kind integral equation derived solely from the Muskhelishvili potential representation. The use of one single representation increases the flexibility of the code and facilitates the incorporation of fast solvers. The fact that the unknown quantity is the limit of an analytic function makes the construction of special corner quadratures easy.

The paper is organized as follows: Section 2 states the stress problem and explains the Muskhelishvili representation. For brevity, finite and infinite domains are treated in the same equations. Section 3 lists relations between the integral operators which form the basis of our scheme. These relations are useful for proving equivalence and uniqueness. Section 4 treats the exterior stress problem for one hole. We compare different integral equations and prove our main result - that a simple Muskhelishvili equation can be constructed. Sections 5 and 6 extend the result of Section 4 to encompass several holes, cracks, and inclusions. The paper ends with Section 7, which gives details about our implementation and presents numerical results. Section 7 reviews a number of small-scale setups. The reason for studying these setups was that we wanted to be confident that our code reproduced previous results before venturing into large-scale examples. As it turned out, some previous results were of poor quality. We were forced to do several independent tests with commercial finite element software to establish correct benchmarks.

\section{PROBLEM STATEMENT AND POTENTIAL REPRESENTATION}

A finite or infinite, linearly elastic, specimen occupies a domain $D$. The outer boundary of the specimen, if it exists, is denoted $\Gamma_{0}$ and is given positive (counter-clockwise) orientation. The domain $D$ is multiply connected. Inside $D$ there are a number $N_{\mathrm{h}}$ of holes and a number $N_{\mathrm{c}}$ of cracks. The holes have boundaries $\Gamma_{j}, j=1,2, \ldots, N_{\mathrm{h}}$, and are given positive orientations. The cracks are denoted $\Gamma_{j}, j=N_{\mathrm{h}}+1, \ldots, N_{\mathrm{h}}+N_{\mathrm{c}}$. The crack $\Gamma_{j}$ starts at crack tip $\gamma_{j \mathrm{~s}}$ and ends at crack tip $\gamma_{j \mathrm{e}}$. The union of all boundaries is $\Gamma$. The left and right sides of $\Gamma$ are distinguished with superscripts $(+)$ and $(-)$. The area of the region enclosed by contour $\Gamma_{j}$ is $A_{j}$. For cracks, it is convenient to choose $A_{j}=-\pi i / 2$. Traction $\left(t_{x}^{\mathrm{pr}}, t_{y}^{\mathrm{pr}}\right)$, that is, stress on the boundary, is prescribed at $\Gamma_{0}^{+}$in the case of a finite domain. Stress $\bar{\sigma}^{\mathrm{pr}}$ is prescribed at infinity in the case of an infinite domain. The holes and the cracks are free of stress. The exterior of the domain is denoted $D^{\prime}$. In passing, we also treat the more general problem of a specimen with $N_{\text {inc }}$ elastic inclusions.

Let $U(x, y)$ denote the Airy stress function. Since $U(x, y)$ satisfies the biharmonic equation everywhere, except for at $\Gamma$, it can be represented as

$$
U(x, y)=\Re \operatorname{e}\{\bar{z} \phi(z)+\chi(z)\}
$$


where the potentials $\phi(z)$ and $\chi(z)$ are possibly multi-valued analytic functions of the complex variable $z=x+i y$. In the stress problem, requiring that the displacements be single-valued, see (12) below, and with certain conditions imposed on the applied external forces, see $(14,15)$ below, $\phi(z)$ and $\chi^{\prime}(z)$ are single-valued, $\chi^{\prime \prime}(z)$ is unique, and $\phi^{\prime}(z)$ is determined up to an imaginary constant in $D$, see Paragraph 40 of Mikhlin [29]. For a thorough discussion of the complex variable approach to elasticity problems, see Refs. [29, 30, 33, 39]. For now, it is sufficient to observe a few relations that link the complex potentials to quantities of physical interest. The traction $t(z)=t_{x}(z)+i t_{y}(z)$ along a curve $\gamma$ is

$$
t(z)=n(z) \Phi(z)+n(z) \overline{\Phi(z)}-\overline{n(z)} z \overline{\Phi^{\prime}(z)}-\overline{n(z) \Psi(z)}
$$

where $\Phi(z)=\phi^{\prime}(z), \Psi(z)=\chi^{\prime \prime}(z)$, and $n(z)=n_{x}(z)+i n_{y}(z)$ is the outward unit normal vector on $\gamma$. The components of the stress tensor in the material are

$$
\begin{gathered}
\sigma_{x x}+\sigma_{y y}=4 \Re \mathrm{e}\{\Phi(z)\} \\
\sigma_{y y}-\sigma_{x x}-2 i \sigma_{x y}=2\left(z \overline{\Phi^{\prime}(z)}+\overline{\Psi(z)}\right) .
\end{gathered}
$$

A natural starting point for stress problems is to represent the potentials $\Phi(z)$ and $\Psi(z)$ in the form of Cauchy-type integrals

$$
\begin{gathered}
\Phi(z)=\frac{1}{2 \pi i} \int_{\Gamma} \frac{\rho \Omega(\tau) \mathrm{d} \tau}{(\tau-z)}+\frac{\alpha}{2}, \quad z \in D \cup D^{\prime}, \\
\Psi(z)=\frac{1}{2 \pi i} \int_{\Gamma} \frac{\rho \Xi(\tau) \mathrm{d} \tau}{(\tau-z)}-\frac{1}{2 \pi i} \int_{\Gamma_{0}} \frac{\bar{n} \overline{t^{\mathrm{pr}}} \mathrm{d} \tau}{(\tau-z)}+\beta, \quad z \in D \cup D^{\prime},
\end{gathered}
$$

where $\Omega(\tau)$ and $\Xi(\tau)$ are unknown layer densities on $\Gamma$, and where $\rho(\tau)$ is a weight which on contours is given by

$$
\rho(\tau)=1, \quad \tau \in \Gamma_{j}, \quad j=0,1, \ldots, N_{\mathrm{h}},
$$

and on the cracks is given by

$$
\rho(\tau)=\left(\left(\tau-\gamma_{j \mathrm{~s}}\right)\left(\tau-\gamma_{j \mathrm{e}}\right)\right)^{-\frac{1}{2}}, \quad \tau \in \Gamma_{j}, \quad j=N_{\mathrm{h}}+1, \ldots, N_{\mathrm{h}}+N_{\mathrm{c}}
$$

In (8) the weight $\rho(\tau)$ is the limit from the right (relative to the orientation of the crack) of the branch given by a branch cut along $\Gamma_{j}$ and

$$
\lim _{\tau \rightarrow \infty} \tau \rho(\tau)=1
$$

Further in $(5,6), t^{\mathrm{pr}}=t_{x}^{\mathrm{pr}}+i t_{y}^{\mathrm{pr}}$ is the prescribed traction at $\Gamma_{0}^{+}$in the case of a finite domain, and the constants $\alpha$ and $\beta$ are related to the stress at infinity $\bar{\sigma}^{\mathrm{pr}}=\left(\sigma_{x x}^{\mathrm{pr}}, \sigma_{y y}^{\mathrm{pr}}, \sigma_{x y}^{\mathrm{pr}}\right)$ in the case of an infinite domain. Three fundamental stresses are $\bar{\sigma}_{\mathrm{I}}^{\mathrm{pr}}=(1,0,0), \bar{\sigma}_{\mathrm{II}}^{\mathrm{pr}}=(0,1,0)$, and $\bar{\sigma}_{\mathrm{III}}^{\mathrm{pr}}=(0,0,1)$. They are obtained by choosing $\alpha=1 / 2$ and $\beta=-1 / 2, \alpha=1 / 2$ and $\beta=1 / 2$, and $\alpha=0$ and $\beta=i$. Note that $\alpha$ always is real and that we have omitted the argument $\tau$ from $n(\tau), \rho(\tau)$, and $t^{\mathrm{pr}}(\tau)$ in $(5,6)$. 
The potential $\Phi(z)$, represented as in (5), will not be unique for the stress problem on a multiply connected domain. As a consequence, we can not expect integral equations derived from this representation to have unique solutions. This is discussed in detail in Paragraph 40 of Mikhlin [29]. Two things need to be fixed. First, the potential $\Phi(z)$ can only be determined up to an imaginary constant inside each contour. By adding a suitable uniqueness condition, for example,

$$
P_{j} \Phi^{+}=0, \quad j=0,1, \ldots, N_{\mathrm{h}},
$$

where $\Phi^{+}$is the limit of $\Phi(z)$ at $\Gamma^{+}$, and where the operator $P_{j}$ is a mapping from $\Gamma_{j}$ to $\mathbb{R}$, defined by

$$
P_{j} f=-\frac{1}{2 A_{j}} \Re \mathrm{e}\left\{\int_{\Gamma_{j}} f(\tau) \bar{\tau} \mathrm{d} \tau\right\}, \quad j=0,1, \ldots, N_{\mathrm{h}},
$$

this indeterminacy is removed. Second, the representation (5) for $\Phi(z)$ may allow for an arbitrary term corresponding to a multi-valued $\phi(z)$ and to multi-valued displacements. By requiring

$$
Q_{j} \rho \Omega=0, \quad j=0,1, \ldots, N_{\mathrm{h}}+N_{\mathrm{c}}
$$

where $Q_{j}$ is a mapping from $\Gamma_{j}$ to $\mathbb{C}$, defined by

$$
Q_{j} f=-\frac{1}{2 A_{j}} \int_{\Gamma_{j}} f(\tau) \mathrm{d} \tau,
$$

in combination with that the applied load satisfies

$$
\begin{aligned}
P_{j} \bar{n} t^{\mathrm{pr}} & =0, \quad j=0,1, \ldots, N_{\mathrm{h}}+N_{\mathrm{c}}, \\
Q_{j} \bar{n} t^{\mathrm{pr}} & =0, \quad j=0,1, \ldots, N_{\mathrm{h}}+N_{\mathrm{c}},
\end{aligned}
$$

we ensure that $\Phi(z)$ has no simple pole inside $\Gamma_{j}$, that $\phi(z)$ is single-valued, that displacements are single-valued, and that the stress problem has a unique solution in terms of $\Phi(z)$ and $\Psi(z)$.

The representations for $\Phi(z)$ and $\Psi(z)$ of $(5,6)$ guarantee that the equations of elasticity are satisfied everywhere in $D \cup D^{\prime}$. The representations also satisfy the boundary conditions at infinity for an infinite domain. It remains only to find $\Omega(\tau)$ and $\Xi(\tau)$ which satisfies the uniqueness conditions $(10,12)$ and the boundary conditions at the holes and at the cracks

$$
\begin{gathered}
t(z)=0, \quad z \in \Gamma_{j}^{-}, \quad j=1,2, \ldots, N_{\mathrm{h}}+N_{\mathrm{c}} \\
t(z)=0, \quad z \in \Gamma_{j}^{+}, \quad j=1,2, \ldots, N_{\mathrm{h}}+N_{\mathrm{c}}
\end{gathered}
$$

and for a finite domain also

$$
\begin{gathered}
t(z)=0, \quad z \in \Gamma_{0}^{-}, \\
t(z)=t^{\mathrm{pr}}, \quad z \in \Gamma_{0}^{+} .
\end{gathered}
$$

If we now demand that the traction jump a quantity $t^{\mathrm{pr}}$ as $\Gamma_{0}$ is crossed and that the traction be continuous as $\Gamma_{j}, j=1,2, \ldots, N_{\mathrm{h}}+N_{\mathrm{c}}$, is crossed, one can express $\Xi(\tau)$ of (6) in 
terms of $\Omega(\tau)$ of (5). The potential $\Psi(z)$ of (6) assumes the form

$$
\begin{gathered}
\Psi(z)=-\frac{1}{2 \pi i} \int_{\Gamma} \frac{\overline{\rho \Omega(\tau)} \mathrm{d} \bar{\tau}}{(\tau-z)}-\frac{1}{2 \pi i} \int_{\Gamma} \frac{\bar{\tau} \rho \Omega(\tau) \mathrm{d} \tau}{(\tau-z)^{2}}-\frac{1}{2 \pi i} \int_{\Gamma_{0}} \frac{\bar{n} \overline{t^{\mathrm{pr}}} \mathrm{d} \tau}{(\tau-z)}+\beta \\
z \in D \cup D^{\prime}
\end{gathered}
$$

A representation of $\psi(z)$, which is related to (20) by partial integration, is the choice of Muskhelishvili for stress problems. See Paragraph 98 of Ref. [30].

\section{OPERATORS AND RELATIONS}

In the following sections we shall frequently use a number of integral operators $M_{1}$, $M_{1}^{*}, M_{1}^{0}$, and $M_{3}$. Here we define these operators, together with some useful relations involving the operators $P_{j}$ and $Q_{j}$ of $(11,13)$.

The Cauchy singular operator $M_{1}$, acting on a function $f(z)$, is given by

$$
M_{1} f(z)=\frac{1}{\pi i} \int_{\Gamma} \frac{f(\tau) \mathrm{d} \tau}{(\tau-z)}, \quad z \in \Gamma
$$

The conjugate of $M_{1}$ is denoted $\overline{M_{1}}$. The part of $M_{1}$ which describes self-interaction, and which is of interests for cracks, is denoted $M_{1}^{*}$

$$
M_{1}^{*} f(z)=\frac{1}{\pi i} \int_{\Gamma_{j}} \frac{f(\tau) \mathrm{d} \tau}{(\tau-z)}, \quad z \in \Gamma_{j}, \quad j=N_{\mathrm{h}}+1, \ldots, N_{\mathrm{h}}+N_{\mathrm{c}}
$$

and the compact remainder of $M_{1}$ is denoted $M_{1}^{0}$

$$
M_{1}^{0} f(z)=\left(M_{1}-M_{1}^{*}\right) f(z), \quad z \in \Gamma_{j}, \quad j=N_{\mathrm{h}}+1, \ldots, N_{\mathrm{h}}+N_{\mathrm{c}} .
$$

The compact operator $M_{3}$ is given by

$$
\begin{gathered}
M_{3} f(z)=\frac{1}{2 \pi i}\left[\int_{\Gamma} \frac{f(\tau) \mathrm{d} \tau}{(\tau-z)}+\frac{\bar{n}}{n} \int_{\Gamma} \frac{f(\tau) \mathrm{d} \tau}{(\bar{\tau}-\bar{z})}\right. \\
\left.+\int_{\Gamma} \frac{\overline{f(\tau)} \mathrm{d} \bar{\tau}}{(\bar{\tau}-\bar{z})}+\frac{\bar{n}}{n} \int_{\Gamma} \frac{(\tau-z) \overline{f(\tau)} \mathrm{d} \bar{\tau}}{(\bar{\tau}-\bar{z})^{2}}\right], \quad z \in \Gamma .
\end{gathered}
$$

The operators $P_{j}$ and $Q_{j}$ satisfy the following relations

$$
\begin{aligned}
P_{j} i & =1, \quad j=0,1, \ldots, N_{\mathrm{h}}, \\
Q_{j} i \bar{z} & =1, \quad j=0,1, \ldots, N_{\mathrm{h}}, \\
P_{j} M_{1} f & =P_{j} M_{3} f, \quad j=0,1, \ldots, N_{\mathrm{h}}, \\
Q_{0} M_{3} f & =-Q_{0} f-\sum_{j=1}^{N_{\mathrm{h}}+N_{\mathrm{c}}} \frac{2 A_{j}}{A_{0}} Q_{j} f, \\
Q_{j} M_{1} f & =-Q_{j} f, \quad j=1,2, \ldots, N_{\mathrm{h}}, \\
Q_{j} M_{3} f & =-Q_{j} f, \quad j=1,2, \ldots, N_{\mathrm{h}},
\end{aligned}
$$


where $f$ is square integrable function. One can, further, show

$$
\begin{aligned}
P_{0} \frac{\bar{n}}{n} \overline{M_{1}} \frac{n}{\bar{n}} f & =-P_{0} f-\sum_{j=1}^{N_{\mathrm{h}}+N_{\mathrm{c}}} \frac{2 A_{j}}{A_{0}} P_{j} f, \\
P_{j} \frac{\bar{n}}{n} \overline{M_{1}} \frac{n}{\bar{n}} f & =-P_{j} f, \quad j=1,2, \ldots, N_{\mathrm{h}}, \\
Q_{0} \frac{\bar{n}}{n} \overline{M_{1}} \frac{n}{\bar{n}} f & =-Q_{0} f-\sum_{j=1}^{N_{\mathrm{h}}+N_{\mathrm{c}}} \frac{2 A_{j}}{A_{0}} Q_{j} f, \\
Q_{j} \frac{\bar{n}}{n} \overline{M_{1}} \frac{n}{\bar{n}} f & =-Q_{j} f, \quad j=1,2, \ldots, N_{\mathrm{h}} .
\end{aligned}
$$

On the cracks we have [16]

$$
\begin{aligned}
Q_{j} \rho & =1, \quad j=N_{\mathrm{h}}+1, \ldots, N_{\mathrm{h}}+N_{\mathrm{c}} \\
Q_{j} \rho M_{1}^{*} \rho^{-1} f & =0, \quad j=N_{\mathrm{h}}+1, \ldots, N_{\mathrm{h}}+N_{\mathrm{c}} \\
M_{1}^{*} \rho M_{1}^{*} \rho^{-1} f(z) & =f(z), \quad z \in \Gamma_{j}, \quad j=N_{\mathrm{h}}+1, \ldots, N_{\mathrm{h}}+N_{\mathrm{c}}, \\
M_{1}^{*} \rho^{-1} M_{1}^{*} \rho f(z) & =f(z)-Q_{j} \rho f, \quad z \in \Gamma_{j}, \quad j=N_{\mathrm{h}}+1, \ldots, N_{\mathrm{h}}+N_{\mathrm{c}} .
\end{aligned}
$$

Finally, we observe that $\Phi(z)^{+}$can be expressed in terms of $M_{1}$ as

$$
\Phi^{+}(z)=\frac{1}{2}\left(I+M_{1}\right) \Omega(z)+\frac{\alpha}{2}, \quad z \in \Gamma,
$$

and that the uniqueness condition (10) can be written as

$$
P_{j}\left(I+M_{1}\right) \Omega=0, \quad j=0,1, \ldots, N_{\mathrm{h}} .
$$

\section{ONE HOLE IN AN INFINITE DOMAIN}

Let us first consider one hole in an infinite domain. There certainly exist classical integral equations for this problem. The most famous may be the Sherman-Lauricella equation, based on the potentials $\phi(z)$ and $\psi(z)$, see Paragraph 56 of Mikhlin [29]. The ShermanLauricella equation does not satisfy $(17,18)$. It also has three undesirable properties: First, it involves the arbitrary placement of points which affects its stability, see Figure 2 of [8] and [13]. Second, it can not easily be extended to setups involving cracks and inclusions. Third, it solves for an unknown density which is related to $\phi(z)$ on $\Gamma$ via the Cauchy singular operator $M_{1}$. This may complicate post-processing [25]. In this section we derive more flexible and efficient integral equations for the hole problem, based on the representations $(5,20)$ for $\Phi(z)$ and $\Psi(z)$. The unknown density is the limiting value of $\Phi(z)$ on $\Gamma$ itself. The representations $(5,20)$ have previously, and successfully, been used in other elastostatic contexts $[13,14,16,40]$. In Sections 5 and 6 we shall extend their use further.

\subsection{A straight-forward integral equation}

Starting with the representations $(5,20)$ for $\Phi(z)$ and $\Psi(z)$, we demand that the traction on $\Gamma$ be zero so that the conditions $(16,17)$ hold. This leads to the integral equation

$$
\left(M_{1}-M_{3}\right) \Omega(z)=\frac{\bar{n}}{n} \bar{\beta}-\alpha, \quad z \in \Gamma,
$$


which has to be solved along with the uniqueness conditions $(12,40)$. This system is not good for numerics. The equation (41) is of Fredholm's first kind and it is not obvious how to implement $(12,40)$. We shall now show that $(12,40,41)$ are equivalent to the following single integral equation of Fredholm's second kind

$$
\left(I-M_{1}\left(M_{3}+i \bar{z} Q_{1}\right)+\frac{i}{2} P_{1}\left(I+M_{1}\right)\right) \Omega(z)=M_{1}\left(\frac{\bar{n}}{n} \bar{\beta}-\alpha\right), \quad z \in \Gamma .
$$

This equation is simply obtained by a linear combination of $(12,40,41)$ and use of that $M_{1}$ is its own inverse.

To show equivalence we apply $M_{1}$ to the left in (42) followed by application of $Q_{1}$ and use of $(26,29,30)$. This gives back (12). Application of $M_{1}$ to the left in (42) and use of (12) followed by application of $P_{1}$ and use of $(25,27)$ gives back $(40)$. Use of $(12,40)$ and application of $M_{1}$ in (42) gives back (41).

\subsection{A Sherman bimaterial type integral equation}

It will be shown in Subsection 7.2 that (42) can lead to very stable numerical algorithms. One drawback is that (42) contains the composition of $M_{1}$ with $M_{3}$. If the integral equations is discretized and solved iteratively, each iterations step will involve two matrixvector multiplications. In this subsection we shall derive an integral equation which is free from this problem. The treatment closely follows Sherman [38]. See also Refs. [9, 16, 40].

Assume that the hole is replaced by an elastic inclusion. The infinite domain $D$ has elastic bulk and shear moduli $\kappa_{1}$ and $\mu_{1}$. The inclusion $D^{\prime}$ has moduli $\kappa_{2}$ and $\mu_{2}$. Sherman [38] derived an integral equation for this problem based on continuity of the integral of traction and of the displacement at $\Gamma$, and on a representation for $\phi(z)$ and $\psi(z)$ related to our representations $(5,20)$ for $\Phi(z)$ and $\Psi(z)$ by partial integration. For our density $\Omega(z)$, Sherman's equation reads

$$
\left(I+d_{1} M_{1}+d_{2} M_{3}\right) \Omega(z)=-d_{1} \alpha-d_{2} \frac{\bar{n}}{n} \bar{\beta}, \quad z \in \Gamma
$$

where the bimaterial parameters $d_{1}$ and $d_{2}$ are given by

$$
\begin{aligned}
& d_{1}=\left(\frac{1}{\kappa_{2}}-\frac{1}{\kappa_{1}}\right) /\left(\frac{1}{\mu_{2}}+\frac{1}{\kappa_{2}}+\frac{1}{\mu_{1}}+\frac{1}{\kappa_{1}}\right), \\
& d_{2}=\left(\frac{1}{\mu_{2}}-\frac{1}{\mu_{1}}\right) /\left(\frac{1}{\mu_{2}}+\frac{1}{\kappa_{2}}+\frac{1}{\mu_{1}}+\frac{1}{\kappa_{1}}\right) .
\end{aligned}
$$

It is easy to see that the solution $\Omega(\tau)$ to (43) satisfies a uniqueness condition similar to (40), namely

$$
P_{1}\left(I+\left(d_{1}+d_{2}\right) M_{1}\right) \Omega=0,
$$

and that the uniqueness condition (12) is satisfied for $d_{1}+d_{2} \neq 1$.

Let now $\kappa_{2}$ and $\mu_{2}$ approach zero in such a way that their ratio is constant. The inclusion has become a hole. We get

$$
d_{1}=\frac{\mu_{2}}{\mu_{2}+\kappa_{2}}
$$




$$
d_{2}=\frac{\kappa_{2}}{\mu_{2}+\kappa_{2}}
$$

and observe that $d_{1}+d_{2}=1$. The uniqueness condition (46) is now the same as (40). The uniqueness condition (12) is no longer satisfied. We therefore replace (43) by

$$
\left(I+d_{1} M_{1}+d_{2} M_{3}+i \bar{z} Q_{1}\right) \Omega(z)=-d_{1} \alpha-d_{2} \frac{\bar{n}}{n} \bar{\beta}, \quad z \in \Gamma,
$$

which can be used both for elastic inclusions and for holes. Application of $Q_{1}$ to the left in $(49)$ and use of $(29,30)$ shows that $(49)$ is the same as $(43)$ and (12). Note that $d_{1}$ is a free parameter. Numerical experiments indicate that the quality of the solution to (49) is rather insensitive to the choice of $d_{1}$. The choice $d_{1}=0.25$ seems to be generally good.

An advantage with (49), over Sherman's original equation [38], is that (49) is based on the potentials $\Phi(z)$ and $\Psi(z)$, simply related to stress via $(3,4)$, while Sherman's original equation is based on the potentials $\phi(z)$ and $\phi(z)$, related to stress via differentiation. We prefer (49) since stress is a quantity of more fundamental interest than displacement in fracture mechanics, and since differentiation is an ill-conditioned numerical operation. Should we wish to obtain displacements, we could always use integration, which is a well-conditioned numerical operation.

\subsection{Muskhelishvili-type integral equations}

The solution $\Omega(z)$ to (42) and to (49) corresponds, because of (10) and (17), to a $\Phi(z)$ which is zero inside the hole $D^{\prime}$. Jump relations in (5) give

$$
\begin{array}{r}
\left(I+M_{1}\right) \Omega(z)=-\alpha, \quad z \in \Gamma, \\
\left(I-M_{1}\right) \Omega(z)=-2 \Phi^{-}(z)+\alpha, \quad z \in \Gamma .
\end{array}
$$

We see, by adding these equations, that $\Omega(z)$ is minus the outside limit of the analytic function $\Phi(z)$ on $\Gamma$. One can therefore view (42) and (49) as an integral equations for $\Phi(z)$.

Fredholm second kind integral equations with $\Phi(z)$ on $\Gamma$ as the unknown variable, with simple (not composed) compact operators on the left hand side, and with possibly singular operators on the right hand side are known as "Muskhelishvili equations". An example is (56), below, for the interior stress problem. Muskhelishvili equations are useful. They solve for the unknown $\Phi(z)$ which is directly related to quantities of physical interest, such as the hydrostatic pressure of (3). Furthermore, on polygonal domains, they allow for the construction of special corner quadratures [14]. The stress in corners has a non-trivial asymptotic behavior, which is remarkably simple to express for $\Phi(z)[14,42]$.

Muskhelishvili equations are considered difficult to construct and to analyze on multiply connected domains. They are therefore not recommended in the classic literature. See $\mathrm{p}$. 398 of Muskhelishvili [30], p. 314 of Sokolnikoff [39], p. 249 and p. 255 of Mikhlin [29], and p. 158 of Parton and Perlin [33]. To illustrate the problem, consider again (41). It is tempting to replace the singular operator $M_{1}$ in (41) by the use of (50) and in this way, somehow, obtain a Fredholm equation. Attempts in this direction easily lead to equations with complicated null-spaces and auxiliary problems may have to be solved. As a consequence, the representation for $\Psi(z)$ of (20) has been rejected in favor of a representation leading to the Sherman-Lauricella equation, whose undesirable properties are listed in the first paragraph of this section.

Perhaps one can say that Sherman's equation of the form (49), for holes, is a Muskhelishvili-type equation? One could argue that the presence of $M_{1}$ on the left hand side dis- 
qualifies (49) from being of Fredholm's second kind. On the other hand, we shall see in Subsection 7.2 that the spectrum of (49) is very similar to the spectrum of the Fredholm second kind equation (42), and that (49) can be implemented almost as stably and efficiently as (42). We therefore conclude that (49) replaces both the Sherman-Lauricella equation and earlier attempts at constructing Muskhelishvili equations.

We now go further and demonstrate that it is possible to derive an equation on multiply connected domains with holes which shares all the properties of the classic Muskhelishvili equation for interior problems (56). We rewrite (43) as

$$
\left(d_{2}\left(I+M_{3}\right)+d_{1}\left(I+M_{1}\right)\right) \Omega(z)=-d_{1} \alpha-d_{2} \frac{\bar{n}}{n} \bar{\beta}, \quad z \in \Gamma .
$$

Then we use (50) and (25), divide by $d_{2}$, and obtain

$$
\left(\left(I+M_{3}\right)+\frac{d_{1}}{d_{2}} P_{1} i\left(I+M_{1}\right)\right) \Omega(z)=-\frac{d_{1}}{d_{2}} \alpha-\frac{\bar{n}}{n} \bar{\beta}, \quad z \in \Gamma .
$$

Adding a term as to satisfy (12) and choosing $d_{1} / d_{2}=1 / 2$ we arrive at

$$
\left(I+M_{3}+\frac{1}{2} P_{1} i\left(I+M_{1}\right)+i \bar{z} Q_{1}\right) \Omega(z)=-\frac{\alpha}{2}-\frac{\bar{n}}{n} \bar{\beta}, \quad z \in \Gamma,
$$

which is the main result of this paper. A proof that (54) has a unique solution is given in the appendix.

We observe that the left hand side of (54) can be evaluated without explicitly computing the action of $M_{1}$ on $\Omega(z)$. Change of the order of integration gives

$$
P_{1} i M_{1} \Omega=-\frac{1}{2 A_{1}} \Re \mathrm{e}\left\{\int_{\Gamma_{1}} \frac{\bar{\tau} \mathrm{d} \tau}{\pi} \int_{\Gamma_{1}} \frac{\Omega(z) \mathrm{d} z}{z-\tau}\right\}=\frac{1}{2 A_{1}} \Re \mathrm{e}\left\{\int_{\Gamma_{1}} \frac{\Omega(z) \mathrm{d} z}{\pi} \int_{\Gamma_{1}} \frac{\bar{\tau} \mathrm{d} \tau}{\tau-z}\right\} .
$$

\section{SEVERAL HOLES OR INCLUSIONS IN INFINITE AND IN FINITE DOMAINS}

Sherman also treats the case of several elastic inclusions in an infinite domain, and in a finite domain [38] with traction applied at $\Gamma_{0}$. Expressed in our quantity $\Omega(z)$, Sherman's equations read

$$
\begin{gathered}
\left(I-M_{3}-2 i P_{0}\right) \rho \Omega(z)=\frac{1}{2}\left(I-\frac{\bar{n}}{n} \overline{M_{1}} \frac{n}{\bar{n}}\right) \bar{n} t^{\mathrm{pr}}, \quad z \in \Gamma_{0}, \\
\left(I+d_{1} M_{1}+d_{2} M_{3}+i \bar{z} Q_{j}\right) \rho \Omega(z)=\frac{d_{2}}{2} \frac{\bar{n}}{n} \overline{M_{1}} \frac{n}{\bar{n}} \bar{n} t^{\mathrm{pr}}-d_{1} \alpha-d_{2} \frac{\bar{n}}{n} \bar{\beta} \\
z \in \Gamma_{j}, \quad j=1,2, \ldots, N_{\mathrm{inc}},
\end{gathered}
$$

where the last term on the left hand side of (57) only is necessary for the special case of an inclusion being a hole, and where the weight $\rho(z)$ of $(7,8)$ has been added as to prepare these equations for the incorporation of cracks, see Section 6 . With the help of $(14,15,25-34)$ it is easy to verify that the uniqueness conditions $(12,40)$ hold for the solution to $(56,57)$. 
For the case of inclusions being holes the corresponding Muskhelishvili equation, which may replace $(57)$, is

$$
\begin{gathered}
\left(I+M_{3}+\frac{1}{2} P_{j} i\left(I+M_{1}\right)+i \bar{z} Q_{j}\right) \rho \Omega(z)=\frac{1}{2} \frac{\bar{n}}{n} \overline{M_{1}} \frac{n}{\bar{n}} \bar{n} t^{\mathrm{pr}}-\frac{\alpha}{2}-\frac{\bar{n}}{n} \bar{\beta} \\
z \in \Gamma_{j}, \quad j=1,2, \ldots, N_{\mathrm{h}} .
\end{gathered}
$$

Remark. Since Sherman [38] does not derive his equations from the potentials $\Phi(z)$ and $\Psi(z)$, but from the potentials $\phi(z)$ and $\psi(z)$, he does not have to worry about our uniqueness condition (12) and he does not use the operators $P_{0}$ and $Q_{j}$. Instead, Sherman introduces other uniqueness conditions related to rigid body displacements and he takes care of them using other operators.

\section{THE PRESENCE OF CRACKS}

It is easy to extend the equations $(56,57,58)$ as to include the presence of cracks. Integral equations for cracks and inclusions (not holes) in an infinite domain, based on the potentials $\Phi(z)$ and $\Psi(z)$ of $(5,20)$, have already been derived in [16]. Integral equations for cracks (only) in a finite domain have been derived in [15]. The extra equations are

$$
\begin{gathered}
\left.\left(I+M_{1}^{*} \rho^{-1}\left(M_{1}^{0} \rho-M_{3} \rho\right)\right)\right) \Omega(z)=-M_{1}^{*} \rho^{-1} \frac{1}{2} \frac{\bar{n}}{n} \overline{M_{1}} \frac{n}{\bar{n}} \bar{n} t^{\mathrm{pr}}+M_{1}^{*} \rho^{-1}\left(\frac{\bar{n}}{n} \bar{\beta}-\alpha\right) \\
z \in \Gamma_{j}, \quad j=N_{\mathrm{h}}+1, \ldots, N_{\mathrm{h}}+N_{\mathrm{c}}
\end{gathered}
$$

Application of $Q_{j}$ to the left in (59) and use of (36) shows that the uniqueness condition (12) holds on the cracks.

\section{NUMERICAL EXAMPLES}

In this section we solve the integral equations $(42,49,54,56,58,59)$ numerically on a SUN Enterprise workstation and study the convergence of computed quantities of physical interest. We cover a wide range of geometries and loads, involving both new and wellstudied setups. We use a Nyström algorithm based on composite Gauss-Legendre and Gauss-Jacobi quadrature and iterative solution. Special quadrature based on Williams basis functions [42] will be used in the corners of finite rectangular domains [14]. A few things can be noted.

- In the examples with holes in an infinite domain we use 16-point Gauss-Legendre quadrature. In the examples with holes and cracks in finite rectangular domains we use 8 -point quadrature. The reason for lowering the order of the quadrature on rectangular domains has to do with stability. It is difficult to get higher-order quadrature stable in the corners.

- The order of the the Nyström scheme will generally be 16 for holes in an infinite domain. The implementation of $M_{3}$ and large parts of $M_{1}$ is 32 nd order, while the implementation of the Cauchy principal value in $M_{1}$, acting on an unknown function, is only 16 th order. In (54) the operator $M_{1}$ is only acting on $\bar{z}$, which is known analytically 
and we can expect full 32 nd order convergence. The asymptotic order for holes and cracks in rectangular domains is approximately 7 . The order of the scheme is limited by the magnitude of the smallest omitted Williams exponent [42] in the special quadrature used on corner panels [14].

- We use a modified uniform mesh on polygonal domains. This means that the outer boundary is first divided into panels of approximately equal lengths in such a way that corners are symmetrically placed in panels. Then some panels, neighboring to corner panels, are made smaller. The reason that we use some a priori refinement is that we aim at very high accuracy and that the solution in the corners is not smooth. See [14] for details.

- The GMRES solver [36] is used for the system of linear equations. The iterations are terminated when the relative norm of the residual is as small as it can get. This often means $10^{-16}$. The number of iterations needed for convergence, given a geometry and a load, is almost independent of the number of discretization points. This is typical for discretized Fredholm integral equations of the second kind.

- The complexity and storage requirement of our implementations for simple geometries grows as $N^{2}$, where $N$ is the total number of discretization points. For the large scale computations in Subsection 7.8 we use the fast multipole method [2, 10, 35], previously used in elastostatic contexts in Refs. [7, 8, 9, 12], and the complexity and the storage requirement is proportional to $N$.

- Great care is devoted to avoiding roundoff error throughout the code. Compensated summation [19, 23] is used for inner products and for matrix-vector multiplications when the fast multipole method is not invoked.

\subsection{Earlier implementations and tests}

Subsets of the integral equations presented above have already been implemented and tested by others and by us. Equation (59), for cracks in an infinite region, has been used for the computation of stress intensity factors and compared to two previous results for a kinked crack [16] and one previous result for a setup involving four straight cracks [12]. The equation has also been used to compute stress intensity factors for spiral-shaped cracks [16] and effective moduli of a periodic array involving ten thousand randomly oriented cracks [12]. Equation (43), for an elastic inclusion in an infinite region was implemented by Theocaris and Ioakimidis [40] and tested on an elliptic inclusion and compared to an analytical solution. Equation (43) and (59) for cracks and inclusions in an infinite region have been tested on a circular inclusion surrounded by 17 straight cracks [16]. Equation (56) for a finite domain has been tested on a starfish shaped region and compared to results obtained by the Sherman-Lauricella equation [13], and also on rectangular domains with V-notches and compared to eight previous results [14]. Equations (56) and (59) for one crack in a finite domain has been tested on a centered crack and compared to 29 previous results [15].

In all the tests mentioned above, where previous results were available, algorithms based on our equations showed dramatic improvement in terms of both stability and economy of points. 

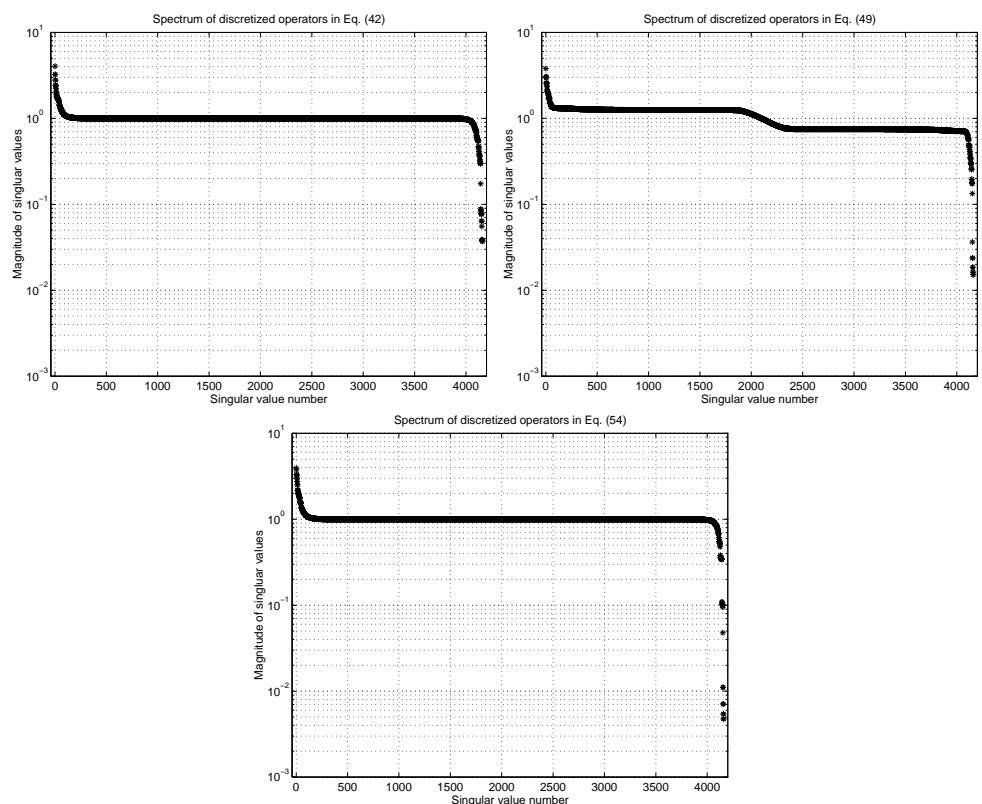

FIG. 1. Singular values for the discretized operators of the left hand side in (42), (49), and (54) for the geometry of (60). A number of 2080 discretization points is used. The condition numbers for the discretized operators are $C_{(42)}=110, C_{(49)}=250$, and $C_{(54)}=830$.

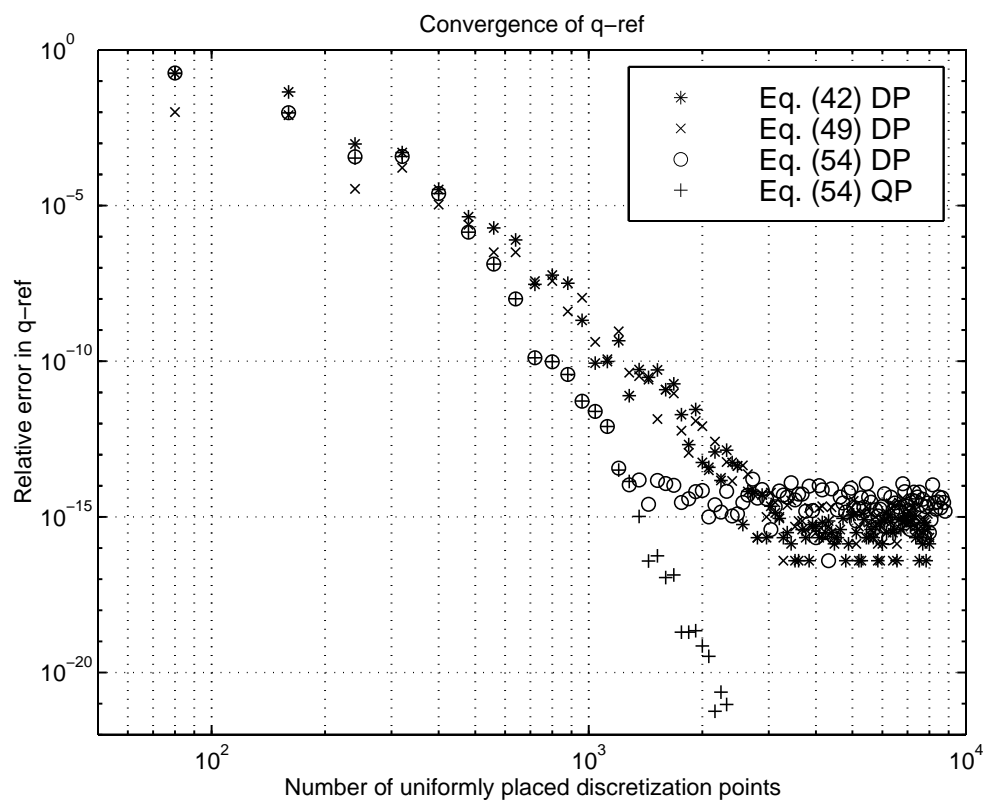

FIG. 2. Convergence of the quantity $q_{\text {ref }}$ of (61) for the hole of (60) and the load at infinity $\bar{\sigma}_{\mathrm{I}}^{\mathrm{pr}}$. Equations (42), (49) and (54) are compared. DP refers to double precision calculations and QP to quadruple precision calculations. The relative errors are computed with the reference quantity taken as $q_{\mathrm{ref}}=5.1445778061927687005756$, which is obtained from (54) with 2400 , or more, points in quadruple precision. A uniform mesh is used, where all quadrature panels have equal lengths in terms of the parameter $t$ of (60). 


\subsection{One hole in an infinite domain}

Let us first consider a nine-armed starfish-shaped hole in an infinite plane parameterized by

$$
z(t)=(1+0.36 \cos 9 t) e^{i t}, \quad 0 \leq t<2 \pi .
$$

The load at infinity is chosen to be $\bar{\sigma}_{\mathrm{I}}^{\mathrm{pr}}=(1,0,0)$, that is, $\alpha=0.5$ and $\beta=-0.5$. We set out to compute a quantity $q_{\text {ref }}$ which is the $L^{2}$ norm of the hydrostatic stress on $\Gamma$

$$
q_{\mathrm{ref}}=\left(\int_{\Gamma}\left(\sigma_{x x}(z)+\sigma_{y y}(z)\right)^{2} \mathrm{~d} s\right)^{\frac{1}{2}}
$$

We shall compare the performance of (42), (49), and (54). Our problem is wellconditioned. Figure 1 depicts the singular values of the discretized operators in the integral equations. The condition number $C$ of the corresponding matrices is approximately $C_{(42)}=110, C_{(49)}=250$, and $C_{(54)}=830$. A convergence study of the quantity $q_{\mathrm{ref}}$ of (61) is presented in Figure 2. The achievable accuracy is better than machine epsilon times the condition numbers of the discretized systems. This is so since the condition number of a system matrix is an upper bound for the condition number of the problem of solving a linear system of equations and since the quantity $q_{\text {ref }}$ computes an average of the solution $\Omega(z)$.

The algorithm based on (42) exhibits the most stable convergence, but it consumes the most computational work per iteration. See Subsection 4.2. The number of GMRES iterations required for full convergence at 4000 discretization points is 31 . The computational work per iteration consumed by algorithms based on (49) and (54) is similar to each other. Computing the action of $M_{1}$ on $\Omega(z)$ can be viewed as a part of the process of computing the action of $M_{3}$ on $\Omega(z)$. However, the result computed by (54) converges faster but less stably, with the number of discretization points, than the results computed by (49). This is so since $M_{1}$ is not acting on $\Omega(z)$ in (54) and since the discretized system (54) has a higher condition number than the discretized system (49). The number of GMRES iterations required for full convergence at 4000 discretization points is 35 with (49) and $d_{1}=0.25$, and 34 with (54). We conclude than the algorithm based on (42) gives the most stable convergence and that it consumes the most computational work. The algorithms based on (54) converges faster than the others and consumes the least work, but it is least stable. The algorithm based on (49) is somewhere in between.

\subsection{One hole in a finite domain}

Let us now consider a circular hole of radius $R$ centered in a rectangular plate with height of $2 h$ and width of $2 w$. Uniform normal traction $t_{y}^{\mathrm{pr}}$ is prescribed at two opposing sides. See Figure 3a. A quantity of interest here is the normalized tangential stress $\hat{\sigma}_{t}(z)$. For a stress-free hole, one can define $\hat{\sigma}_{t}(z)$ as the ratio of the trace of the stress tensor on the hole to the trace of the applied stress tensor. The relation for the present setup becomes

$$
\hat{\sigma}_{t}(z)=\frac{\sigma_{x x}(z)+\sigma_{y y}(z)}{t_{y}^{\mathrm{pr}}}, \quad z \in \Gamma_{1} .
$$



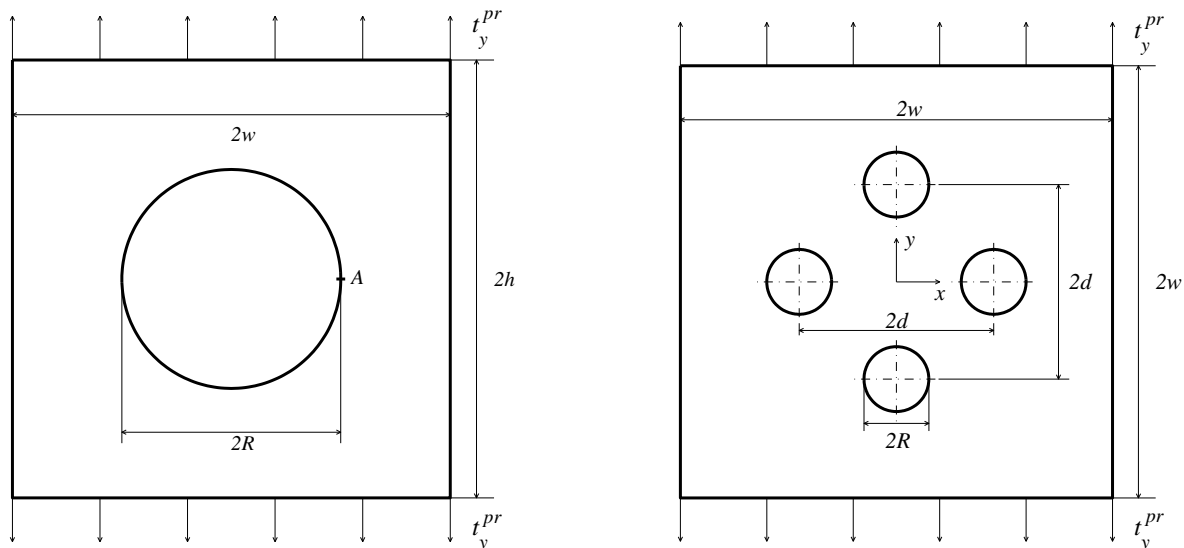

FIG. 3a. Left, a hole of radius $R$ centered in a rectangular plate with height $2 h$ and width $2 w$. A uniaxial stress is applied at two opposing sides. The remaining two sides are stress-free. A maximum absolute value of the normalized tangential stress (63) will occur at point $A$.

FIG. 3b. Right, four holes of radius $R$ in a square plate with side-length $2 w$. The hole are symmetrically placed on the coordinate axes, a distance $d$ from the origin.

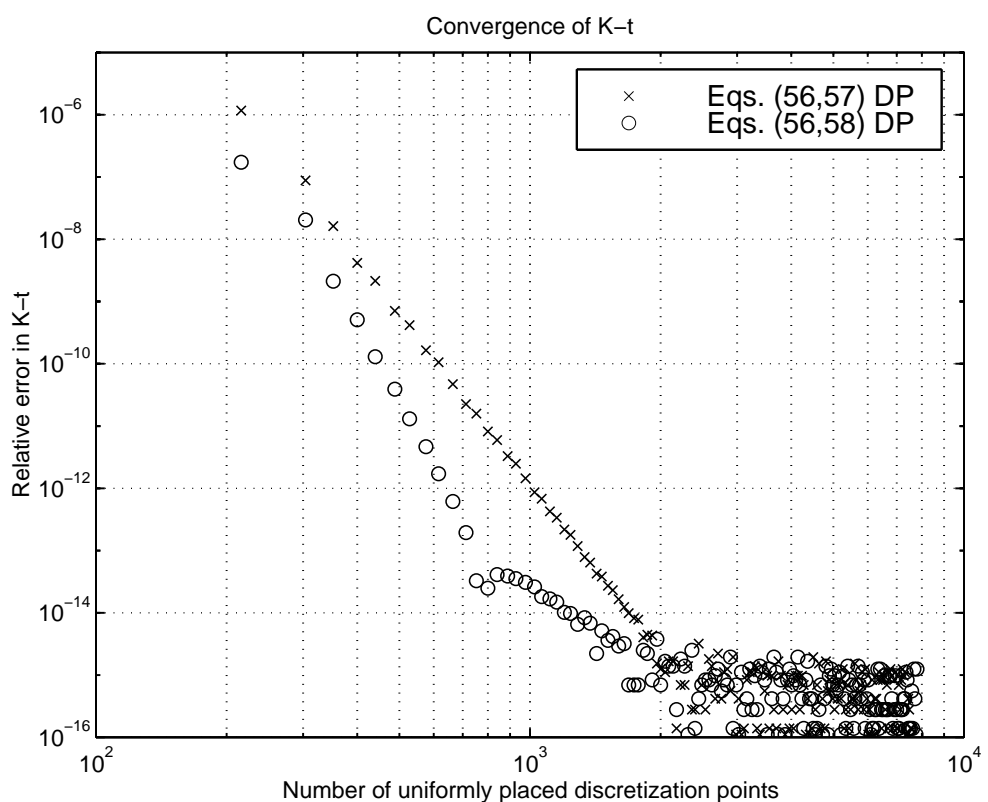

FIG. 4. Convergence of the stress concentration factor $K_{t}$ of (63) for a circular hole of radius $R=0.25$, centered in a square plate of unit side-length. A uniform uniaxial load applied. The systems $(56,57)$, and $(56,58)$ are compared in double precision arithmetic. The problem is well-conditioned. The relative errors are computed with a reference value taken as $K_{t}=6.3886960194568237$. A modified uniform mesh is used, where all quadrature panels have approximately equal lengths.

A stress concentration factor $K_{t}$ can be defined as the maximum absolute value of the normalized tangential stress

$$
K_{t}=\max _{z \in \Gamma_{1}}\left|\hat{\sigma}_{t}(z)\right|
$$

The value of $K_{t}$ for this setup, with $R / w=0.5$ and $h / w=1$, has previously been computed by Isida and Sato [21], with a method based on series expansions, and by Nisitani 
and Chen [31] with a method based on singular integral equations. The authors report the values $K_{t}=6.3887$ and $K_{t}=6.38869$, respectively. Figure 4 compares the performance of the two systems $(56,57)$, and $(56,58)$ for this setup. Our computations converge to a value of $K_{t}=6.3886960194568$, which is reached at about 2000 discretization points. With only 216 points, which corresponds to the coarsest mesh we can use without violating the rules for the construction our modified uniform mesh [14], we get $K_{t}=6.38870$ and confirm the result of the previous authors. This computation takes only a few seconds.

Figure 4 shows that the convergence rate of the algorithm based on the systems $(56,57)$ is approximately eighth order. It is controlled by the operator $M_{1}$ on the left hand side of (57). The convergence of the algorithm based on the system $(56,58)$ is initially faster, since $M_{1}$, thanks to (55), does not have to be evaluated on the left hand side of (58). With more than 800 points, the error related to the accuracy of the corner quadrature dominates. It is seventh order. It is worth mentioning that for the setup of Figure 3a, the location of the point $z$, where the normalized tangential stress has its maximum, is known in advance and that we arrange the mesh so that a quadrature point is placed there. If we were to perform the maximization of (63) numerically, the order of both schemes would decrease.

As for convergence in the GMRES iterative solver, the algorithms based on the two systems are rather similar. The algorithms based on $(56,57)$ requires no more than 30 GMRES iterations for full convergence, while the algorithm based on $(56,58)$ requires no more than 28 iterations, irrespective of the number of discretization points used.

The value for $K_{t}$ of the setup in Figure 3a has also been computed for the case $R / w=0.1$ and $h / w=1$, by Leung, Zavareh, and Beskos [26] and for the case $R / w=0.5$ and $h / w=10$ by Chen, Ting, and Yang [4]. Leung, Zavareh, and Beskos [26] use a variety of methods, including a combined finite element/boundary element scheme, and the commercial software BEASY and NASTRAN. The authors exploit symmetry, use a number of 40 to 100 points for a quarter of the geometry, and arrive at computed values lying in the range $K_{t}=2.889$ to $K_{t}=3.059$. With our coarsest mesh, corresponding to 176 discretization points for the entire geometry, we get the estimate $K_{t}=3.0860851$. Full accuracy is achieved at about 600 points and the converged value is $K_{t}=3.0860851670536$. Since the discrepancy between our value and those of Ref. [26] is rather large considering the simple nature of the geometry, we asked Dr. Jonas Faleskog at the Department of Solid Mechanics, KTH, to do an independent investigation with the commercial finite element package ABAQUS. Using bi-quadratic elements and a number of 24576 degrees of freedom for a quarter of the geometry, Dr. Faleskog obtained the estimate $K_{t}=3.0861 \pm 0.0001$. This computation took about ten seconds, the time for mesh generation not included, and confirms our result. Chen, Ting, and Yang [4] use a combination of a boundary element method and a spectral method and arrive at a value of $K_{t}=4.32$ for their choice of parameters. A number of 32 to 96 quadratic boundary elements on an adaptive mesh and 12 Fourier modes were used. With a number of 360 discretization points, corresponding to the coarsest uniform mesh we can construct, we compute the estimate $K_{t}=4.347$. Full accuracy is achieved at about 2000 points and the converged value is $K_{t}=4.3475991016650$. A possible explanation to the discrepancy between our results and those of Ref. [4] can be found in the convergence study in Table 1 of Ref. [4]. This table indicates that 12 Fourier modes is not enough to guarantee three accurate digits. 

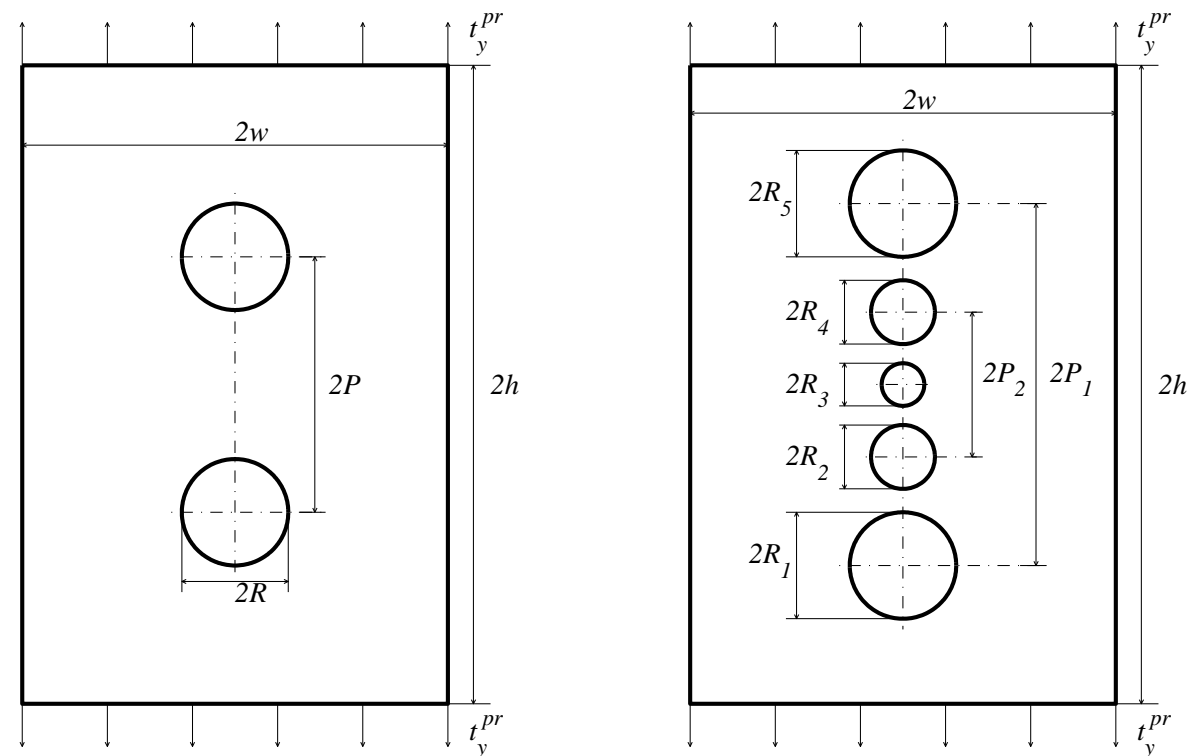

FIG. 5a. Left, two symmetrically placed holes of radius $R$ separated a distance $2 P$ in a rectangular plate with height $2 h$ and width $2 w$. A uniaxial stress is applied at two opposing sides. The remaining two sides are stress-free.

FIG. 5b. Right, five symmetrically placed holes.

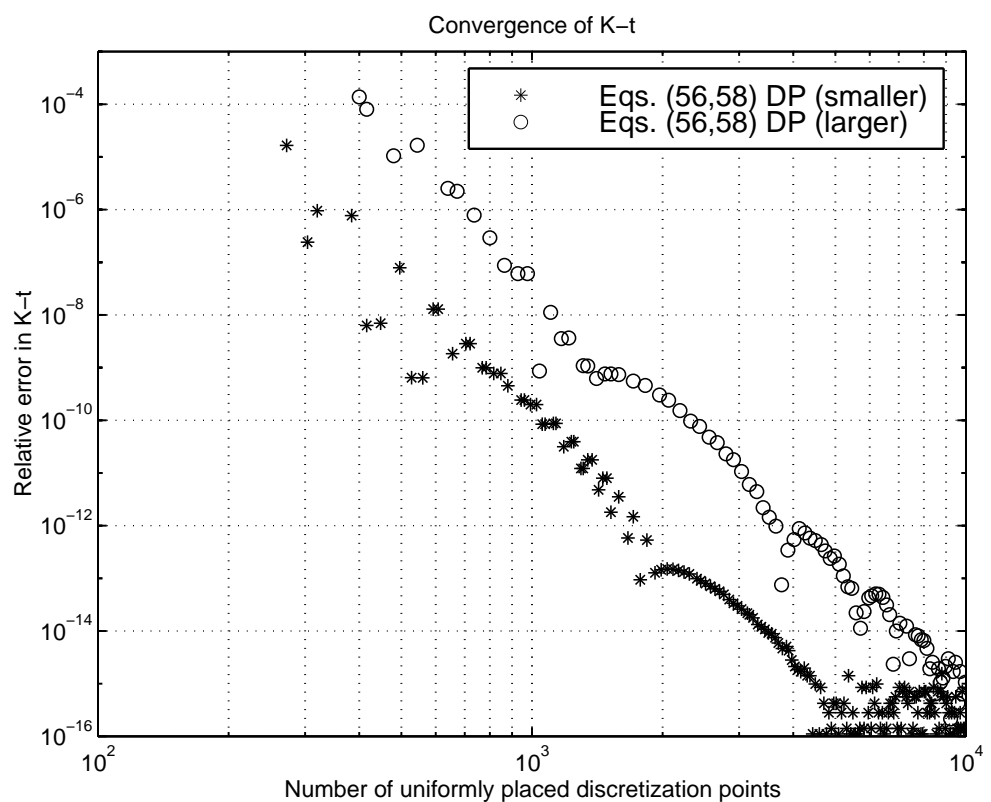

FIG. 6. Convergence of the stress concentration factor $K_{t}$ of (63) for the setup of Figure 5a. The system $(56,58)$ is used in double precision arithmetic. Stars refer to a setup with smaller holes given by $R / w=$ $0.25, P / w=5 / 6$, and $h / w=3.125$. Open circles refer to a setup with larger holes given by $R / w=0.5$, $P / w=1$, and $h / w=10$. The reference values for the two cases are taken as $K_{t}=3.1471561193558794$ and $K_{t}=4.1693288541234566$, respectively. A modified uniform mesh is used. 


\subsection{Two holes in a finite domain}

One setup studied by Chen, Ting, and Yang [4] involves two equisized holes of radius $R / w=0.5$ separated a distance $P / w=1$ in a rectangle with $h / w=10$, see Figure 5a. A number of 30 to 60 quadratic elements and a number of 12 to 24 Fourier modes on each hole were used. The result converged to $K_{t}=4.17$, which coincides with the estimate of Atsumi [1] for a specimen with $h / w=\infty$. We used the algorithm based on the system $(56,58)$ for this setup. A convergence study is presented in Figure 6 . In this problem, the location of the point $z$ on $\Gamma$ where $\hat{\sigma}_{t}(z)$ of (62) achieves its maximum is not known in advance, and we have to interpolate the solution to $(56,58)$ in order to find the maximum. The interpolation is eighth order accurate. The optimization is done with Newton's method and safeguarded with Golden Section Search. This procedure slows down the rate of convergence compared to the case of a single, centered hole. Still, as can be seen in Figure 6, with 400 discretization points, we get the result $K_{t}=4.17$ which confirms the results of the previous investigators. The computations converge to a value $K_{t}=4.16932885412$, which is reached at about 6000 points.

Another, simpler, setup studied by Chen, Ting, and Yang [4], involves two smaller equisized holes of radius $R / w=0.25$ separated a distance $P / w=5 / 6$ in a rectangle with $h / w=3.125$, see Figure 5a. Here the authors compare their own result $K_{t}=3.145$ with a result $K_{t}=3.139$ obtained with the commercial finite element software ANSYS using 2132 elements, and a result $K_{t}=3.345$ obtained by Meguid [28] with the finite element package SUPERB. With only 272 discretization points we compute the four digit accurate answer $K_{t}=3.147$, see Figure 6 . With 3300 , or more, points we get the converged value $K_{t}=3.1471561193559$.

\subsection{Four holes in a finite domain}

Woo and Chan [41] studied 28 setups of square plates centered at the origin and aligned with the coordinate axes of a cartesian coordinate system. The plates had side-lengths $2 w$ and contained four small holes with radii varying from $R / w=0.01$ to $R / w=0.15$. The hole centers were placed at the four points $(d, 0),(0, d),(-d, 0)$, and $(0,-d)$, where $d$ varied between $d / R=2$ and $d / R=5$. The applied load was uniaxial. See Figure 3b. A collocation method based on series expansions for the potentials $\Phi(z)$ and $\Psi(z)$, adaptive placement of the collocation points, and least squares approximation was used. The number of degrees of freedom used corresponds to 320 for the entire geometry. Results for $K_{t}$ of (63) were presented to four digits in the authors' Table 2.

We checked all 28 results for $K_{t}$ of Woo and Chan [41] against results obtained using $(56,58)$. For 22 setups we confirm all their four digits, for four setups we confirm three digits, for one setup we confirm two digits, and for one setup we do not confirm a single digit. The discrepancies between our results and those of Woo and Chan [41] chiefly took place for the most difficult setup with the largest holes, see Table 1 . The very large discrepancy for the setup with $d / R=5$ deserves some comment. Not only do our results differ from those of Woo and Chan [41] with 20 per cent. Further, while Woo and Chan [41] find that the maximum stress concentration occurs on the holes centered around $(0, d)$ and $(0,-d)$ we find that the maximum occurs at the holes centered around $(d, 0)$ and $(-d, 0)$. For this reason we decided to perform computations with the commercial finite element software ANSYS. We used about 10000 eight-node quadratic elements of the type PLANE82 corresponding to about 31000 discretization points per configuration. The results of these computations are presented in Table 1. 
TABLE 1

Results for the stress concentration factor $K_{t}$ of $(63)$ for the most challenging setups of four holes in a square plate of Woo and Chan [41].

\begin{tabular}{lllll}
\hline$d / R^{*}$ & Ref. [41] & ANSYS $^{a}$ & Eqs. $(56,58)$ and 320 pts $^{b}$ & Eqs. $(56,58)$ best poss. $^{c}$ \\
\hline 2 & 4.833 & $4.832 \pm 0.001$ & 4.8327 & 4.8326684347317 \\
2.5 & 4.332 & $4.331 \pm 0.001$ & 4.332 & 4.3314395844773 \\
3 & 4.051 & $4.050 \pm 0.001$ & 4.051 & 4.0504940219307 \\
3.5 & 3.814 & $3.813 \pm 0.001$ & 3.814 & 3.8142986073521 \\
4 & 3.768 & $3.769 \pm 0.001$ & 3.7694 & 3.7693606053913 \\
4.5 & 3.860 & $3.941 \pm 0.001$ & 3.942 & 3.9414430052749 \\
5 & 3.905 & $4.764 \pm 0.001$ & 4.765 & 4.7639166341954 \\
\hline
\end{tabular}

* The plate has side length $2 w$ and the hole radii are $R / w=0.15$. The holes are placed on the coordinate axes a distance $d$ from the origin.

$a$ ANSYS refers to finite element computations.

$b$ Results from $(56,58)$ using only 320 discretization points.

${ }^{c}$ Best possible results in double precision arithmetic from $(56,58)$.

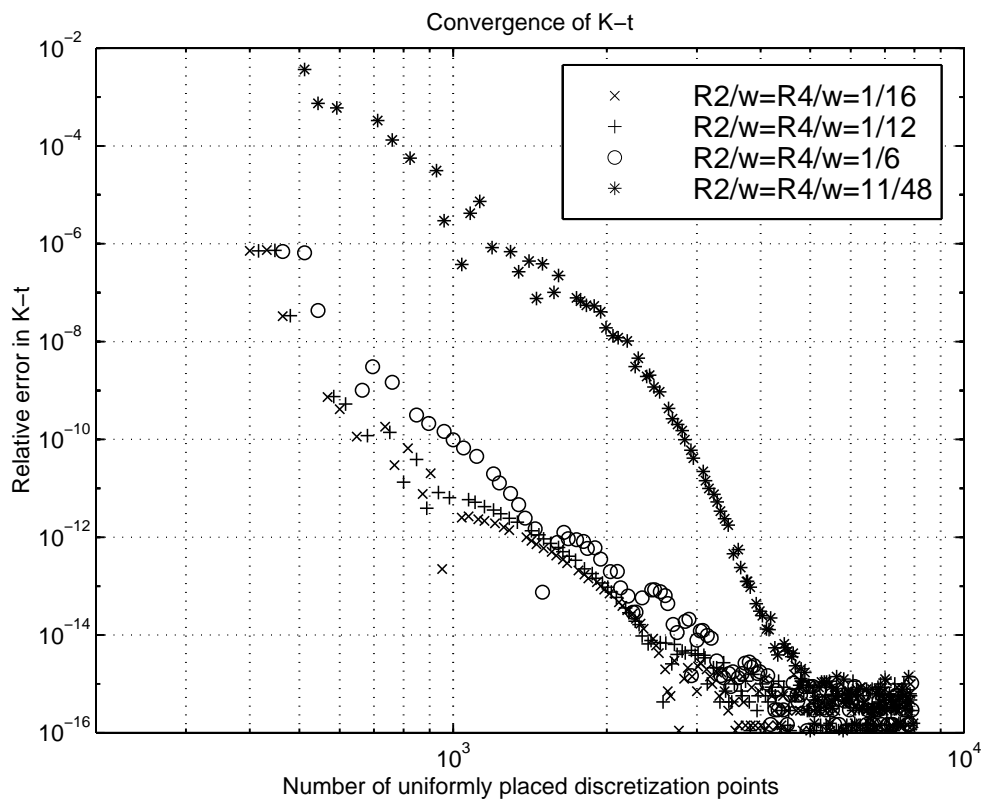

FIG. 7. Convergence of the stress concentration factor $K_{t}$ of (63) for the five hole setup of Figure 5b. The system $(56,58)$ is used in double precision arithmetic. The plate has a height-to-width ratio of $h / w=3.125$. The two holes furthest removed from the origin have radii $R_{1} / w=R_{5} / w=0.25$. The hole at the origin has radius $R_{3} / w=1 / 16$. The separation distances are $P_{1} / w=5 / 6$ and $P_{2} / w=2 / 6$. The symbols 'x', ' + ', 'o', and '*' refer to radii $R_{2}=R_{4}=1 / 16, R_{2}=R_{4}=1 / 12, R_{2}=R_{4}=1 / 6$, amd $R_{2}=R_{4}=11 / 48$. The reference values are taken as $K_{t}=3.1191735564211897, K_{t}=3.1069156838873726, K_{t}=2.9969770267128966$, and $K_{t}=2.8309208646076457$, respectively.

\subsection{Five holes in a finite domain}

The most complex hole system in a finite rectangular plate that we could find results for in the literature is the system denoted "condition (III)" by Meguid [28] and Chen, Ting, and Yang [4]. This system involves five small symmetrically aligned holes in a plate with $h / w=3.125$. The two holes furthest removed from the origin have radii $R_{1} / w=R_{5} / w=0.25$. The hole at the origin has radius $R_{3} / w=1 / 16$. The radii of the remaining two holes are allowed to vary between $R_{2}=R_{4}=1 / 16$ and $R_{2}=R_{4}=11 / 48$. 
TABLE 2

Results for the stress concentration factor $K_{t}$ of $(63)$ of Figure $5 b$.

\begin{tabular}{cllll}
\hline$R_{2}=R_{4}^{*}$ & Ref. [4] BEAM & Ref. [4] ANSYS & Ref. [28] SUPERB & Eqs. (56,58) \\
\hline$w / 16$ & 3.115 & 3.110 & 3.244 & 3.1191735564212 \\
$w / 12$ & 3.102 & 3.095 & 3.204 & 3.1069156838874 \\
$w / 6$ & 2.980 & 2.974 & 3.049 & 2.9969770267129 \\
$11 w / 48$ & 2.792 & 2.790 & 2.981 & 2.8309208646076 \\
\hline
\end{tabular}

* Three radii are fixed: $R_{1} / w=R_{5} / w=0.25$ and $R_{3} / w=1 / 16$. The radii $R_{2}$ and $R_{4}$ vary. The separation distances are $P_{1} / w=5 / 6$ and $P_{2} / w=2 / 6$.

The separation distances are $P_{1} / w=5 / 6$ and $P_{2} / w=2 / 6$, see Figure $5 \mathrm{~b}$. The stress concentration factor for the two outer holes reported by the previous authors, as well as our new results are displayed in Table 2. The mesh is not completely uniform. The spacing between discretization points is taken four times denser on the holes than on the outer boundary. The reason for this a priori refinement is that the holes are much closer to each other than to the outer boundary.

It can be noted, in Table 2 that the newer results of Chen, Ting, and Yang [4] are generally better than the older results of Meguid [28]. The largest discrepancies are found for the most difficult case where $R_{2}=R_{4}=11 / 48$. Here the holes are closest to each other.

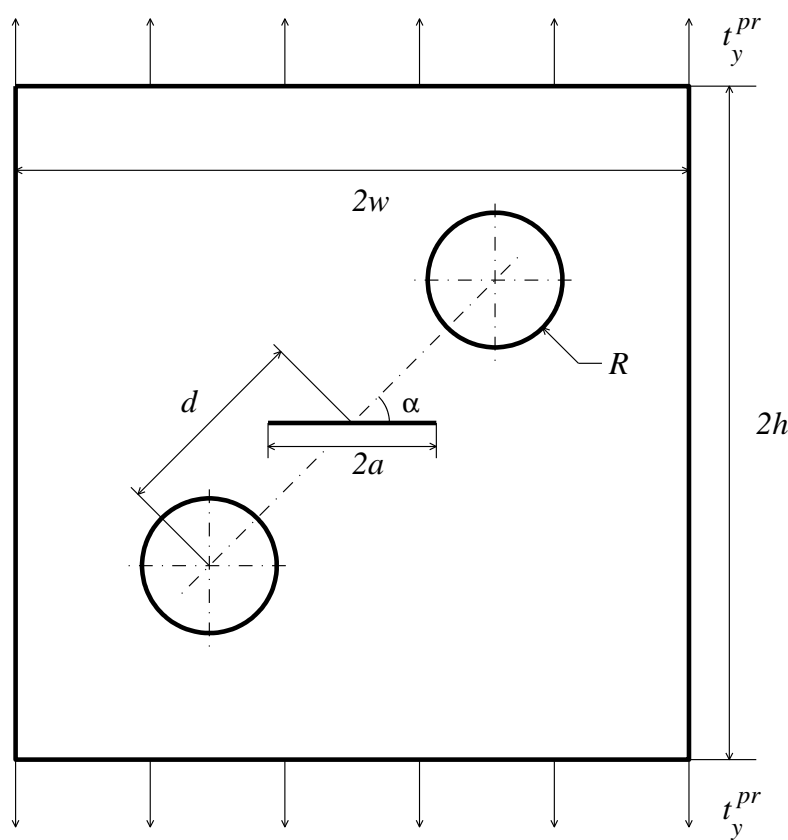

FIG. 8. Two holes of radii $R$ and a crack of length $2 a$ in a square plate with side-length $2 w$. The distance from the hole-centers to the origin is $d$. The angle of a line through the hole-centers and the $x$-axis is $\alpha$.

\subsection{Holes and cracks in a finite domain}

Let us now consider setups involving equisized holes of radii $R$ and straight cracks of length $2 a$. A quantity, of particular interest in this context is the complex valued normalized 
stress intensity factor $F=F_{\mathrm{I}}+i F_{\mathrm{II}}$, which can be computed as

$$
\begin{gathered}
F\left(\gamma_{\mathrm{s}}\right)=\frac{i \sqrt{2}}{t_{y}^{\mathrm{pr}} \sqrt{a}} \lim _{z \rightarrow \gamma_{j \mathrm{~s}}} \overline{\Omega\left(\gamma_{\mathrm{s}}\right) \rho(z)} \sqrt{\delta s(z)}, \quad z \in \Gamma_{j}, \quad j=N_{\mathrm{h}}+1, \ldots, N_{\mathrm{h}}+N_{\mathrm{c}}(64) \\
F\left(\gamma_{\mathrm{e}}\right)=-\frac{i \sqrt{2}}{t_{y}^{\mathrm{pr}} \sqrt{a}} \lim _{z \rightarrow \gamma_{\mathrm{j}}} \overline{\Omega\left(\gamma_{\mathrm{e}}\right) \rho(z)} \sqrt{\delta s(z)}, \quad z \in \Gamma_{j}, \quad j=N_{\mathrm{h}}+1, \ldots, N_{\mathrm{h}}+N_{\mathrm{c}}
\end{gathered}
$$

where $\delta s(z)$ is arclength measured from the closest crack tip.

Woo and Chan [41] studied 23 setups involving two small holes and one small straight crack in a square plate with side-length $2 w$ and centered at the origin. The crack is placed at the origin and aligned with the $x$-axis. The two holes are placed opposite each other and at a distance $d$ from the origin. The angle between a line through the holes and the $x$-axis is $\alpha$. See Figure 8. The setup is such that $a / w=0.1, d / w=0.3, R / w=0.1$, and $\alpha$ is allowed to vary. Numerical results for $F_{\mathrm{I}}$ and $F_{\mathrm{II}}$ are presented to four or to five digits.

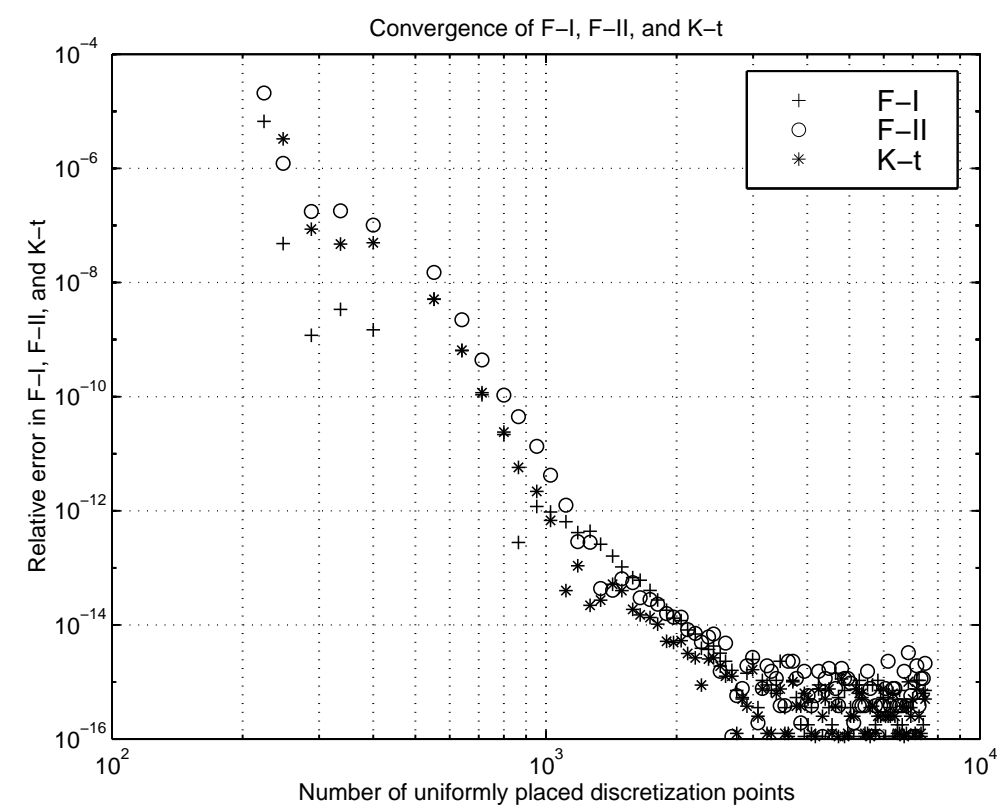

FIG. 9. Convergence of the stress concentration factor $K_{t}$ of (63) and the normalized stress intensity factor $F_{\mathrm{I}}$ and $F_{\mathrm{II}}$ of $(64,65)$ for the setup in of Figure 8 . The system $(56,58,59)$ is used in double precision arithmetic. The two holes have radii $R / w=0.1$. The distance from the origin is $d / w=0.3$. The angle of inclination is $\alpha=\pi / 4$. The crack has length $a / w=0.1$. The reference values are taken as $K_{t}=3.5220865511473008$, $F_{\mathrm{I}}=1.2503879831741171$, and $F_{\mathrm{II}}=-0.14416713067935393$, respectively. The mesh is uniformly refined. The discretization points are placed four times closer to each other on the cracks and on the holes than on the outer boundary.

With $\alpha=0$ and $\alpha=\pi / 2$ and with our equations $(56,58,59,64,65)$ we reproduce the results of Woo and Chan [41] for $F_{\mathrm{I}}$ to all digits presented in their Table 6 . The value of $F_{\mathrm{II}}$ is zero due to the symmetry. For all other values of the angle $\alpha$ we get results for $F_{\mathrm{I}}$ and $F_{\text {II }}$ that differ significantly from those in Table 6 of Woo and Chan [41]. The mismatch is particularly severe for $F_{\mathrm{II}}$, where we sometimes do not even get the same sign as Woo and Chan [41]. As an example we take $\alpha=\pi / 4$. Here Woo and Chan [41] report $F_{\mathrm{I}}=1.2532$ and $F_{\mathrm{II}}=0.4039$ in their Table 6 (and $F_{\mathrm{I}}=1.2530$ and $F_{\mathrm{II}}=0.4045$ in their Table 5), 
while we get convergence to $F_{\mathrm{I}}=1.2503879831741$ and $F_{\mathrm{II}}=-0.1441671306794$, see Figure 9. Again, we performed computations with the commercial finite element software ANSYS and about 10000 eight-node quadratic elements of the type PLANE82, to validate our code. Near the crack tips, a focused mesh with quarter point triangular elements was used. The stress intensity factors were obtained through a fit of the nodal displacements near the crack tip to the asymptotic analytical crack tip solution. The result from ANSYS was $F_{\mathrm{I}}=1.250 \pm 0.003$ and $F_{\mathrm{II}}=-0.14 \pm 0.01$, which indicates that our code based on $(56,58,59)$ is implemented correctly.

\subsection{Large scale computations}

In a final example we solve for stress concentration factors $K_{t}$ and normalized stress intensity factors $F_{\mathrm{I}}$ and $F_{\mathrm{II}}$ for some large setups using equations $(56,58,59,63,64,65)$ and the fast multipole method. We choose a simply reproducible setup consisting of a square plate of side length $2 w$ with $2 m^{2}$ equisized circular holes of radii $R$ and $2 m^{2}$ straight cracks of length $2 a$. The total number of object is thus $4 \mathrm{~m}^{2}$. The size of the objects are chosen as $R / w=a / w=0.25 / \mathrm{m}$ and the objects are placed on square grid with a distance $w / \mathrm{m}$ between nearest grid points. See Figure 10. In particular, we study the convergence of $K_{t}$ and the largest value $F_{\mathrm{I}}$ and $F_{\mathrm{II}}$ with the size of the setup, determined by the parameter $m$, and with the number of uniformly placed discretization points $N$.
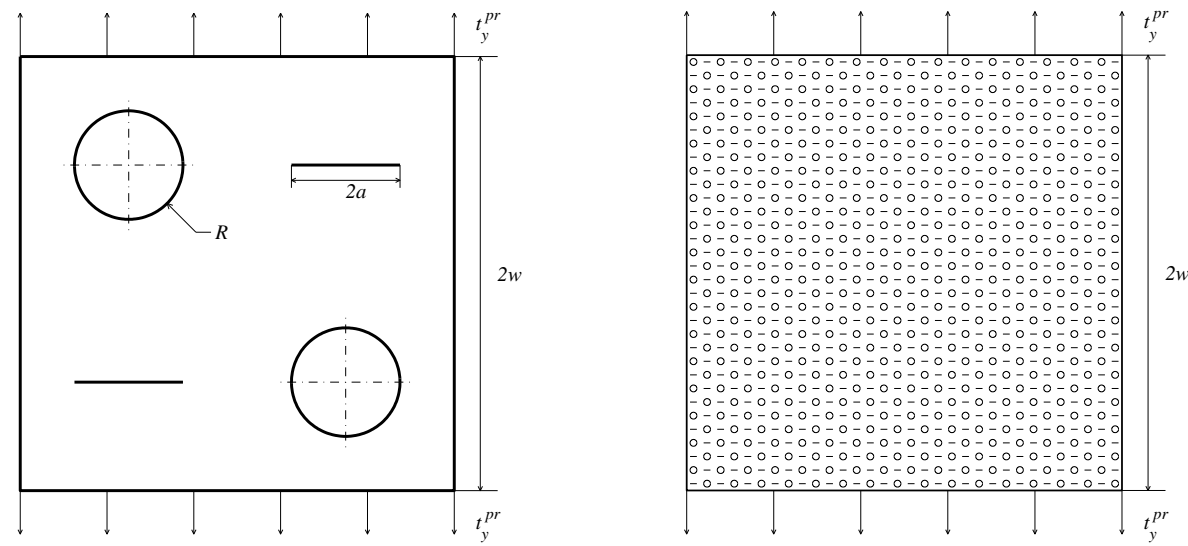

FIG. 10. Two setups with holes and cracks. The square plates have side lengths $2 w$ and contain $2 m^{2}$ circular holes with radii $R=0.25 \mathrm{w} / \mathrm{m}$ and $2 \mathrm{~m}^{2}$ cracks of length $2 a=0.5 \mathrm{w} / \mathrm{m}$. The objects are placed on square grid with a distance $w / m$ between nearest grid points. The left plate has $m=1$ and the right plate has $m=16$.

As it turns out, the largest tangential stress and the largest stress intensity factors are always found on cracks and holes closest to the plate corners. In Table 3 we show how these quantities converge with the parameter $m$. The largest value of $m$ chosen is $m=32$, corresponding to 4096 objects. Figure 11 shows convergence of $K_{t}$ along with the largest values of $F_{\mathrm{I}}$ and $F_{\mathrm{II}}$ for a plate with $m=16$, that is, with 1024 objects. As can be seen, the achievable accuracy for this large-scale computation is about three digits less than in the examples of the preceding subsections. The chief reason for this loss of accuracy is not that our large-scale example is more ill-conditioned than the previous examples, but due to numerical cancellation in the fast multipole scheme as differences between positions of the discretization points are evaluated. In the smaller examples of the preceding subsections these differences were computed with special care. 
TABLE 3

Results for the stress concentration factor $K_{t}$ of (63) and largest normalized stress intensity factors $F_{\mathrm{I}}$ and $F_{\mathrm{II}}$ of $(64,65)$ in the setup of Figure 10.

\begin{tabular}{rlll}
\hline$m^{*}$ & $K_{t}^{a}$ & $F_{\mathrm{I}}^{a}$ & $F_{\mathrm{II}}^{a}$ \\
\hline 1 & 4.748140332912 & 1.133105345114 & -0.059347687764 \\
2 & 4.7341318940 & 1.1442488313 & -0.0545599210 \\
4 & 4.7339636409 & 1.1441818139 & -0.0542844102 \\
8 & 4.7340021110 & 1.1441832610 & -0.0542907947 \\
16 & 4.734000002 & 1.144183308 & -0.054290737 \\
32 & 4.733998851 & 1.144183030 & -0.054290181 \\
\hline
\end{tabular}

* The number of holes and cracks is $4 m^{2}$.

$a$ Equations $(56,58,59)$ are used with a modified uniform mesh.

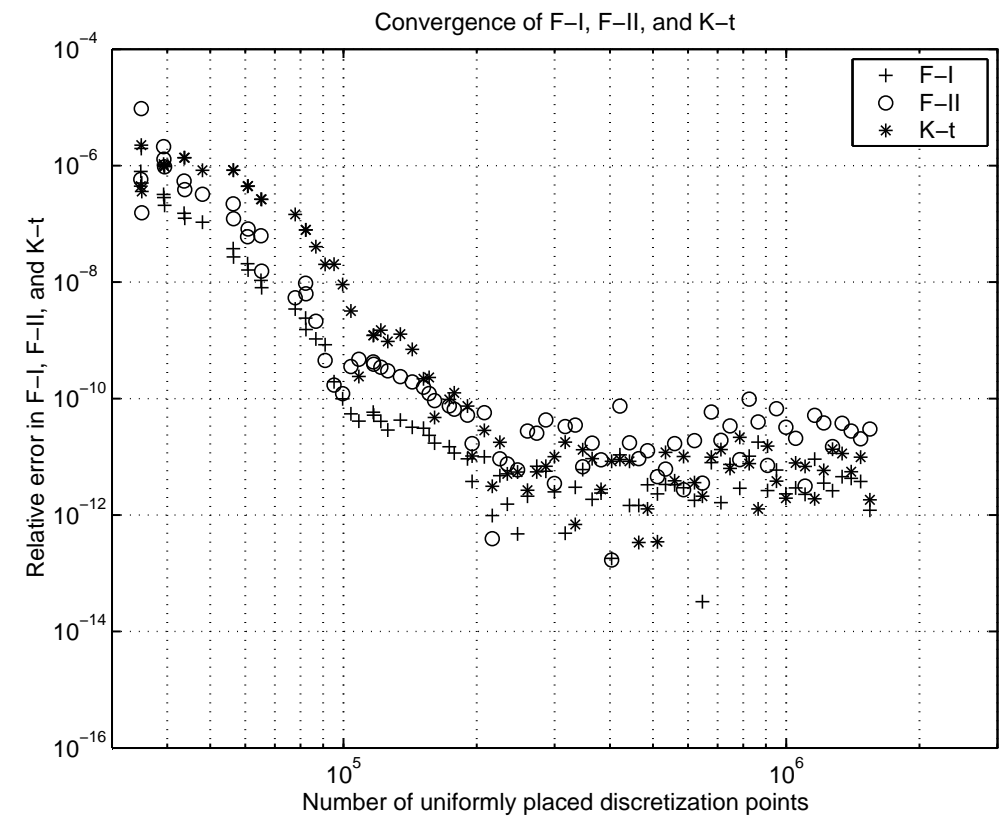

FIG. 11. Convergence of the stress concentration factor $K_{t}$ of (63) and the largest normalized stress intensity factor $F_{\mathrm{I}}$ and $F_{\mathrm{II}}$ of $(64,65)$ for the setup in of Figure 10 with $m=16$, corresponding to 1024 holes and cracks. The system $(56,58,59)$ is used in double precision arithmetic and the fast multipole method is used for matrixvector multiplication. The reference values are taken as $K_{t}=4.7340000015921, F_{\mathrm{I}}=1.1441833078687$, and $F_{\mathrm{II}}=-0.054290736731657$. The mesh is uniformly refined. About 50 GMRES iterations are needed for full convergence.

\section{CONCLUSION AND DISCUSSION}

We derived the Muskhelishvili-type integral equation (54) for a multiply connected domain with a hole. The equation is simple. It does not require the solution of any "auxiliary problems for some particular types of loadings" as has been the case for previous equations of this type according to p. 158 of Ref. [33]. The analysis of the equation seems, in our opinion, not more complicated than the analysis of the classic Sherman-Lauricella equation. The difficulty of the analysis of Muskhelishvili-type equations is the reason why the Sherman-Lauricella should be preferred, according to the authors referenced in Subsection 4.3. Undesirable properties of the Sherman-Lauricella equation, avoided in our formulation, are listed in the first paragraph of Section 4. 
The equation (54) is not the only integral equation for multiply connected domains which can be derived from the Muskhelishvili potential representation. Equations (42) and (49) are two other examples, each with their own advantages and drawbacks. The reason that we prefer (54) over (42) and (49) has to do with speed and convergence. Further, one could construct modifications of (54) by replacing the operator $P_{j}$ with some other operator which has the property (25). The reason that we prefer $P_{j}$ in (54) has to do with simplicity. The operator $P_{j}$ naturally appears in (14) and we want to stick to a small number of operators. While $P_{j}$ may not be an optimal choice, we believe it is sufficiently good for our purposes.

Interestingly, it is recommended that crack problems, which involve multiply connected domains, should be solved with algorithms based on the Muskhelishvili potential representation. See Paragraph 23 in Ref. [33] and Section 6 of Chapter V in Ref. [34]. In $(56,58,59)$ we unite these results and get a set of equations for bodies containing an arbitrary number of cracks, holes, and inclusions. These equations have several properties which are good for numerics, especially so in the context of polygonal domains. Here, too, minor modifications are possible. For example, by changing the uniqueness condition (10), which is somewhat arbitrarily chosen, alternative equations can be derived. Anyhow, in a series of small-scale examples we greatly improved on previous benchmarks. It was easy to review a large number of setups and results, since our algorithm is comparatively flexible. We saw that commercial finite element packages often performed better than special purpose codes for these simple problems.

Finally, we solved some large but well-conditioned problems. The largest problem studied involved 4096 objects, that is, on the order of 1000 times more objects than the typical research paper in this field. A slightly smaller problem was resolved in double precision arithmetic using 250000 uniformly placed discretizations points and overresolved with up to 1550000 points as to demonstrate stability. Naturally, as the number of discretization points increases the achievable accuracy goes down. As a rule of thumb, we get about $16-\log _{10} N$ correct digits, where $N$ is the number of discretization points needed for resolution. It is our hope that this paper will encourage more reliable calculations in the field of computational fracture mechanics. We also believe our work to have relevance for the problem of Stokes flow, multicomponent fluid flow, and other large-scale multiphase problems in materials science where similar equations are to be solved [8, 20, 24, 25] and where the Sherman-Lauricella equation and equations based on primitive variables do not perform to satisfaction unless preconditioners are used $[8,20]$.

\section{APPENDIX}

We shall show that the homogeneous equation (54) has no non-trivial solutions. Using relations in Section 3 and

$$
P_{1} i M_{3} f=-P_{1} i f
$$

it is easy to show that the homogeneous version of equation (54)

$$
\left(I+M_{3}+\frac{1}{2} P_{1} i\left(I+M_{1}\right)+i \bar{z} Q_{1}\right) \Omega(z)=0, \quad z \in \Gamma,
$$

is equivalent to the following four equations

$$
\left(I+M_{3}\right) \Omega(z)=0, z \in \Gamma,
$$




$$
\begin{aligned}
P_{1} i\left(I+M_{1}\right) \Omega & =0, \\
Q_{1} \Omega & =0, \\
P_{1}\left(I+M_{1}\right) \Omega & =0 .
\end{aligned}
$$

Application of $Q_{1}$ to the left in (A.2) gives (A.5). Subtraction of (A.5) from (A.2) and multiplication by $i$, followed by application of $P_{1}$ gives (A.4). Subtraction of (A.5) and (A.4) from (A.2) gives (A.3). Equation (A.6) follows from the application of $P_{1}$ to (A.3). A linear combination of (A.3-A.5) gives back (A.2).

We shall focus on (A.3) and show that any non-trivial solution $\Omega_{0}(z)$ to this equation satisfies

$$
\left(I+M_{1}\right) \Omega_{0}(z)=c_{1}, \quad z \in \Gamma,
$$

where $c_{1}$ is a real-valued constant. If $c_{1}$ is non-zero, this violates (A.4). If $c_{1}$ is zero, then a substitution of (A.7) in (A.3) gives that $\Omega_{0}(z)$ must satisfy

$$
\left(M_{1}-M_{3}\right) \Omega_{0}(z)=0,
$$

which is the homogeneous version of (41). Under the uniqueness condition (A.5), there is only one non-trivial solution to (A.8), namely $\Omega_{0}(z)=i c_{2}$, where $c_{2}$ is another real-valued constant. If $c_{2}$ is non-zero, this violates (A.6).

It remains to show (A.7). For this we introduce new potentials in D

$$
\Phi^{*}\left(z^{\prime}\right)=-\frac{1}{2 \pi} \int_{\Gamma} \frac{\Omega_{0}(\tau) \mathrm{d} \tau}{\left(\tau-z^{\prime}\right)} \quad z^{\prime} \in D
$$

and

$$
\Psi^{*}\left(z^{\prime}\right)=\frac{1}{2 \pi} \int_{\Gamma} \frac{\overline{\Omega_{0}(\tau)} \mathrm{d} \bar{\tau}}{\left(\tau-z^{\prime}\right)}+\frac{1}{2 \pi} \int_{\Gamma} \frac{\bar{\tau} \Omega_{0}(\tau) \mathrm{d} \tau}{\left(\tau-z^{\prime}\right)^{2}} . \quad z^{\prime} \in D,
$$

Taking limits from the inside of $D$, one can show

$$
\lim _{z^{\prime} \rightarrow z \in \Gamma} n \Phi^{*}\left(z^{\prime}\right)+n \overline{\Phi^{*}\left(z^{\prime}\right)}-\bar{n} z^{\prime} \overline{\Phi^{*^{\prime}\left(z^{\prime}\right)}}-\bar{n} \overline{\Psi^{*}\left(z^{\prime}\right)}=i n\left(I+M_{3}\right) \Omega_{0}(z) .
$$

Equation (A.11) implies that a solution $\Phi^{*}\left(z^{\prime}\right)$ to

$$
\lim _{z^{\prime} \rightarrow z \in \Gamma} n \Phi^{*}\left(z^{\prime}\right)+n \overline{\Phi^{*}\left(z^{\prime}\right)}-\bar{n} z \overline{\Phi^{*^{\prime}}\left(z^{\prime}\right)}-\bar{n} \overline{\Psi^{*}\left(z^{\prime}\right)}=0,
$$

corresponds, via (A.9), to a density $\Omega_{0}(z)$ which is a non-trivial solution to (A.3). According to Paragraph 34 of Muskhelishvili [30], the only solution to (A.11) is $\Phi^{*}\left(z^{\prime}\right)=i c_{3}$, where $c_{3}$ is another real-valued constant. Taking the limit $z^{\prime} \rightarrow z \in \Gamma$ from the inside of $D$ in (A.9) we see that

$$
\left(I+M_{1}\right) \Omega_{0}(z)=-2 c_{3}, \quad z \in \Gamma
$$

and with $c_{1}=-2 c_{3}$ this proves (A.7). 


\section{REFERENCES}

1. A. Atsumi, On the stresses in a strip under tension and containing two equal circular holes placed longitudinally, ASME Journal of Applied Mechanics 23, 555-562 (1956).

2. J. Carrier, L. Greengard, and V. Rokhlin, A fast adaptive multipole algorithm for particle simulations, SIAM Journal on Scientific and Statistical Computing 9, 669-686 (1988).

3. K. T. Chau and Y. B. Wang, A new boundary integral formulation for plane elastic bodies containing cracks and holes, International Journal of Solids and Structures 36, 2041-2074 (1999).

4. K. T. Chen, K. Ting, and W. S. Yang, Stress analysis of two-dimensional perforated plates using boundary element alternating method, Computers and Structures 75, 515-527 (2000).

5. C.-S. Chen, R. Krause, R. G. Pettit, L. Banks-Sills, and A. R. Ingraffea, Numerical assessment of T-stress computation using a $p$-version finite element method, International Journal of Fracture 107, 177-199 (2001).

6. C. Daux, N. Moës, J. Dolbow, N. Sukumar, and T. Belytschko, Arbitrary branched and intersecting cracks with the extended finite element method, International Journal for Numerical Methods in Engineering 48, 1741-1760 (2000)

7. A. Greenbaum, L. Greengard, and A. Mayo, On the numerical solution of the biharmonic equation in the plane, Physica D 60, 216-225 (1992).

8. L. Greengard, M. C. Kropinski, and A. Mayo, Integral equation methods for Stokes flow and isotropic elasticity in the plane, Journal of Computational Physics 125, 403-414 (1996), doi:10.1006/jcph.1996.0102.

9. L. Greengard and J. Helsing, On the numerical evaluation of elastostatic fields in locally isotropic twodimensional composites, Journal of the Mechanics and Physics of Solids 46, 1441-1462 (1998).

10. L. Greengard and V. Rokhlin, A fast algorithm for particle simulations, Journal of Computational Physics 73 , 325-348 (1987).

11. L. Gu, A compatible boundary element method for plane elasticity and fracture mechanics, Applied Mathematical Modelling 17, 394-405 (1993).

12. J. Helsing, Fast and accurate numerical solution to an elastostatic problem involving ten thousand randomly oriented cracks, International Journal of Fracture 100, 321-327 (1999).

13. J. Helsing, On the interior stress problem for elastic bodies. ASME Journal of Applied Mechanics 67, 658-662 (2000)

14. J. Helsing and A. Jonsson, On the computation of stress fields on polygonal domains with V-notches, International Journal for Numerical Methods in Engineering, 53, 433-453 (2002), doi:10.1002/nme.291.

15. J. Helsing and A. Jonsson, A seventh order accurate and stable algorithm for the computation of stress inside cracked rectangular domains, Department of Solid Mechanics KTH Research Report TRITA-HLF-294, 2001.

16. J. Helsing and G. Peters, Integral equation methods and numerical solutions of crack and inclusion problems in planar elastostatics, SIAM Journal on Applied Mathematics 59, 965-982 (1999).

17. J. Helsing and G. Peters, An efficient numerical algorithm for cracks partly in frictionless contact, SIAM Journal on Applied Mathematics 61, 551-566 (2000).

18. S.-H. Her, Fracture analysis of interfacial crack by global-local finite element, International Journal of Fracture 106, 177-193 (2000)

19. N. J. Higham, Accuracy and stability of numerical algorithms (SIAM, Philadelphia, 1996) pp. 92-97.

20. T. Y. Hou, J. S. Lowengrub, and M. J. Shelley, Boundary integral methods for multicomponent fluids and multiphase materials, Journal of Computational Physics 169, 302-362 (2001), doi:10.1006/jcph.2000.6626.

21. M. Isida and R. Sato, Analysis of finite regions containing a circular hole or inclusion with application to their periodic arrays, Transactions of the Japan Society of Mechanical Engineers A 50, 1619-1627 (1984).

22. M. A. Jaswon and G. T. Symm, Integral equation methods in potential theory and elastostatics (Academic Press, London, 1977).

23. W. Kahan, Further remarks on reducing truncation errors, Communications of the Association for Computing Machinery 8, 40 (1965).

24. M. C. A. Kropinski, Integral equation methods for particle simulation in creeping flows, Computers \& Mathematics with Applications 38, 67-87 (1999).

25. M. C. A. Kropinski, An Efficient Numerical Method for Studying Interfacial Motion in Two-Dimensional Creeping Flows, Journal of Computational Physics (in the press 2001).

26. K. L. Leung, P. B. Zavareh, and D. E. Beskos, 2-D elastostatic analysis by a symmetric BEM/FEM scheme, Engineering Analysis with Boundary Elements 15, 67-78 (1995). 
27. Y. J. Liu, N. Xu, and J. F. Luo, Modeling of interphases in fiber-reinforced composites under transverse loading using the boundary element method, ASME Journal of Applied Mechanics 67, 41-49 (2000).

28. S. A. Meguid, Finite element analysis of defence hole systems for the reduction of stress concentration in a uniaxially-loaded plate with two coaxial holes, Engineering Fracture Mechanics 25, 403-413 (1986).

29. S. G. Mikhlin, Integral equations (Pergamon Press, London, 1957).

30. N. I. Muskhelishvili, Some Basic Problems of the Mathematical Theory of Elasticity (P. Noordhoff Ltd, Groningen, 1953).

31. H. Nisitani and D. H. Chen, Body force method and its applications to numerical and theoretical problems in fracture and damage, Computational Mechanics 19, 470-480 (1997).

32. A. Portela, M. H. Aliabadi, and D. P. Rooke, The dual boundary element method: effective implementation for crack problems, International Journal for Numerical Methods in Engineering 33, 1269-1287 (1992).

33. V. Z. Parton and P. I. Perlin, Integral Equation Methods in Elasticity, (MIR, Moscow, 1982).

34. V. Z. Parton and P. I. Perlin, Mathematical Methods of the theory of Elasticity (MIR, Moscow, 1984).

35. V. Rokhlin, Rapid solution of integral equations of classical potential theory, Journal of Computational Physics 60, 187-207 (1985).

36. Y. Saad and M. H. Schultz, GMRES: a generalized minimum residual algorithm for solving nonsymmetric linear systems, SIAM Journal on Scientific and Statistical Computing 7, 856-869 (1986).

37. A. Sáez, R. Gallego, and J. Domínguez, Hypersingular quarter-point boundary elements for crack problems, International Journal for Numerical Methods in Engineering 38, 1681-1701 (1995).

38. D. I. Sherman, On the problem of plane strain in non-homogeneous media, in Non-homogeneity in Elasticity and Plasticity, edited by W. Olszak, (Pergamon Press, 1959) pp. 3-20.

39. I. S. Sokolnikoff, Mathematical Theory of Elasticity (McGraw-Hill, New York, 1956).

40. P. S. Theocaris and N. I. Ioakimidis, The inclusion problem in plane elasticity, Quarterly Journal of Mechanics and Applied Mathematics 30, 437-448 (1977).

41. C. W. Woo and W. S. Chan, Boundary collocation method for analyzing perforated plate problems, Engineering Fracture Mechanics 43, 757-768 (1992).

42. M. L. Williams, Stress singularities resulting from various boundary conditions in angular corners of plates in extension, ASME Journal of Applied Mechanics 19, 526-528 (1952).

43. Q. Z. Xiao, B. L. Karilahoo, and F. W. Williams, Application of penalty-equilibrium hybrid stress element method to crack problems, Engineering Fracture Mechanics 63, 1-22 (1999). 